\title{
14. GEOCHEMISTRY OF CARBONATE NODULES AND CEMENTS AND IMPLICATIONS FOR HYDROTHERMAL CIRCULATION, MIDDLE VALLEY, JUAN DE FUCA RIDGE ${ }^{1}$
}

\author{
Paul A. Baker, ${ }^{2}$ Scott L. Cross, ${ }^{2}$ and Stephen J. Burns ${ }^{3}$
}

\begin{abstract}
Carbonate cements and nodules from hydrothermal sediments of Middle Valley were analyzed for their mineralogical, chemical, and isotopic compositions. These diagenetic carbonates include calcite, high-magnesium calcite, and dolomite. The chemical sources for carbonate precipitation include alteration of basement rocks (calcium and strontium), diffusion from seawater (magnesium), recrystallization of biogenic calcite (calcium and carbon), oxidation of sedimentary organic matter (carbon), and oxidation of thermogenic methane (carbon).

The shallowest diagenetic carbonates from Hole $857 \mathrm{C}$ that were analyzed are dolomites. These are succeeded downcore by high-magnesium calcites and, finally, low-magnesium calcites. The shallowest diagenetic carbonates from Hole 858D are highmagnesium calcites which are succeeded downcore by low-magnesium calcites. Dolomites also occur in a limited depth zone in Hole 858D. These dolomites have a seawater magnesium source, but their calcium must be solely derived from basement-derived hydrothermal sources.

Temperature gradients calculated from oxygen isotopic compositions of the carbonates are $0.53^{\circ} \mathrm{C} / \mathrm{m}$ in Hole $857 \mathrm{C}$ and $10.5^{\circ} \mathrm{C} / \mathrm{m}$ in Hole $858 \mathrm{D}$. The distribution of temperatures is consistent with conductive heat transfer over the analyzed sections.

We hypothesize from chemical data that Hole $858 \mathrm{D}$ intersected a fault zone at $28 \mathrm{~m}$ below the seafloor. The fault zone is believed to be a major conduit for the hydrothermal fluids discharging at the nearby vent. The dissolved $\mathrm{CO}_{2}$ in this fluid has a calculated carbon isotopic composition of about $-20 \%$ Peedee belemnite (PDB), implying its source from oxidation of sedimentary organic carbon. A small fraction of the hydrothermal fluid advects laterally at a sub-bottom depth of 3 to $7 \mathrm{~m}$. This flow is not rapid enough to significantly affect the heat transport, but it does affect the mineralogical, chemical (iron and manganese contents), and isotopic compositions of the diagenetic carbonates.
\end{abstract}

\section{INTRODUCTION}

Young, sediment-covered seafloor spreading centers have rarely been sampled by deep-sea drilling. Systematic efforts to study such environments by deep drilling have been made only on Deep Sea Drilling Project (DSDP) Leg 64 in the Guaymas Basin of the Gulf of California, DSDP Leg 23 in the Red Sea, and Ocean Drilling Program (ODP) Leg 139 in Middle Valley of the northern Juan de Fuca Ridge (Fig. 1). Although this type of ridge represents a relatively minor proportion of all active spreading centers, the geological importance of this setting can hardly be overemphasized; all trailing edge continental margins evolved from sediment-covered spreading centers. Likewise, many of the world's great petroleum and sulfide ore accumulations originated in this type of sedimentary environment.

The primary goals of drilling on Leg 139 were to understand the nature of hydrothermal circulation at a sediment-covered ridge and to attempt to link hydrothermal circulation to the origin of sedimenthosted massive sulfide ore bodies. This chapter addresses several aspects of both goals. Specifically, we discuss the thermal record preserved in the sediments and the contributions of various sources to the chemistry and isotopic composition of hydrothermal solutions.

Unfortunately, it is difficult to frame this discussion in a context of geological history. One of the frustrations of Leg 139 was that both the magnetostratigraphic and biostratigraphic records were poorly preserved. Future studies in this region must seek to improve our ability to determine geological ages of important events.

This study relies on analyses of one component of the sediments: alkaline earth carbonates. We have studied these carbonates in three of the four sites on ODP Leg 139 (Fig. 2): Site 856, near a fossil sulfide

\footnotetext{
${ }^{1}$ Mottl, M.J., Davis, E.E., Fisher, A.T., and Slack, J.F. (Eds.), 1994. Proc. ODP, Sci. Results, 139: College Station, TX (Ocean Drilling Program).

${ }_{2}^{2}$ Duke University, Department of Geology, Durham, NC 27708, U.S.A.

${ }^{3}$ Universitat Bern, Geologisches Institut, Baltzerstrasse 1, CH-3012, Bem, Switzer-
}

ore deposit; Site 857, a hydrothermal "reservoir" site; and Site 858, adjacent to or within an active hydrothermal discharge zone. We present information about the occurrence and mineralogy of diagenetic carbonates, the stable isotopic and strontium isotopic compositions of these carbonates, and the major and minor elemental compositions of these carbonates. Many of our conclusions are, necessarily, preliminary. Further refinements and modelling await more detailed analyses of the carbonates, associated sediments, and pore waters.

\section{OCCURRENCE OF CARBONATE SEDIMENTS, NODULES, AND CEMENTS}

Alkaline earth carbonates (predominantly calcite and dolomite) are an important component of sediments recovered during Leg 139 . They occur in a variety of forms. Biogenic calcite, predominantly foraminifers and coccoliths, is the major source of sedimentary carbonate in most holes. Because of the high rates of detrital sedimentation, the carbonate content of sediments at all sites seldom exceeds 10 weight percent (wt\%) as $\mathrm{CaCO}_{3}$ (Davis, Mottl, Fisher, et al., 1992; Baker and Cross, this volume). The highest values are found near the tops of holes in sediments of latest Pleistocene or Holocene age which, uniformly across Middle Valley, are finer-grained, more pelagic, and less detrital than older sediments.

Biogenic calcite is rapidly recrystallized to diagenetic calcite downcore as a consequence of the high thermal gradients in Middle Valley sediments. In Hole $855 \mathrm{C}$ calcite fossils persist to greater than 100 mbsf. Hole $856 \mathrm{~A}$ is barren of foraminifers and nannofossils below about $50 \mathrm{mbsf}$; Hole $856 \mathrm{~B}$ is barren below about $40 \mathrm{mbsf}$; Holes $857 \mathrm{~A}$ and $857 \mathrm{C}$ have poor calcareous microfossil preservation below 83 mbsf; Hole 858A is barren below 111 mbsf; Hole 858B is barren below 16 mbsf; Hole $858 \mathrm{C}$ below $41 \mathrm{mbsf}$; and Hole $858 \mathrm{D}$ is barren below about 29 mbsf (Davis, Mottl, Fisher, et al., 1992). The disappearance of calcareous microfossils deep in various holes is generally correlative with lower present-day thermal gradients in those holes. The most obvious exceptions are Holes $856 \mathrm{~A}$ and $856 \mathrm{~B}$. Evidence presented 


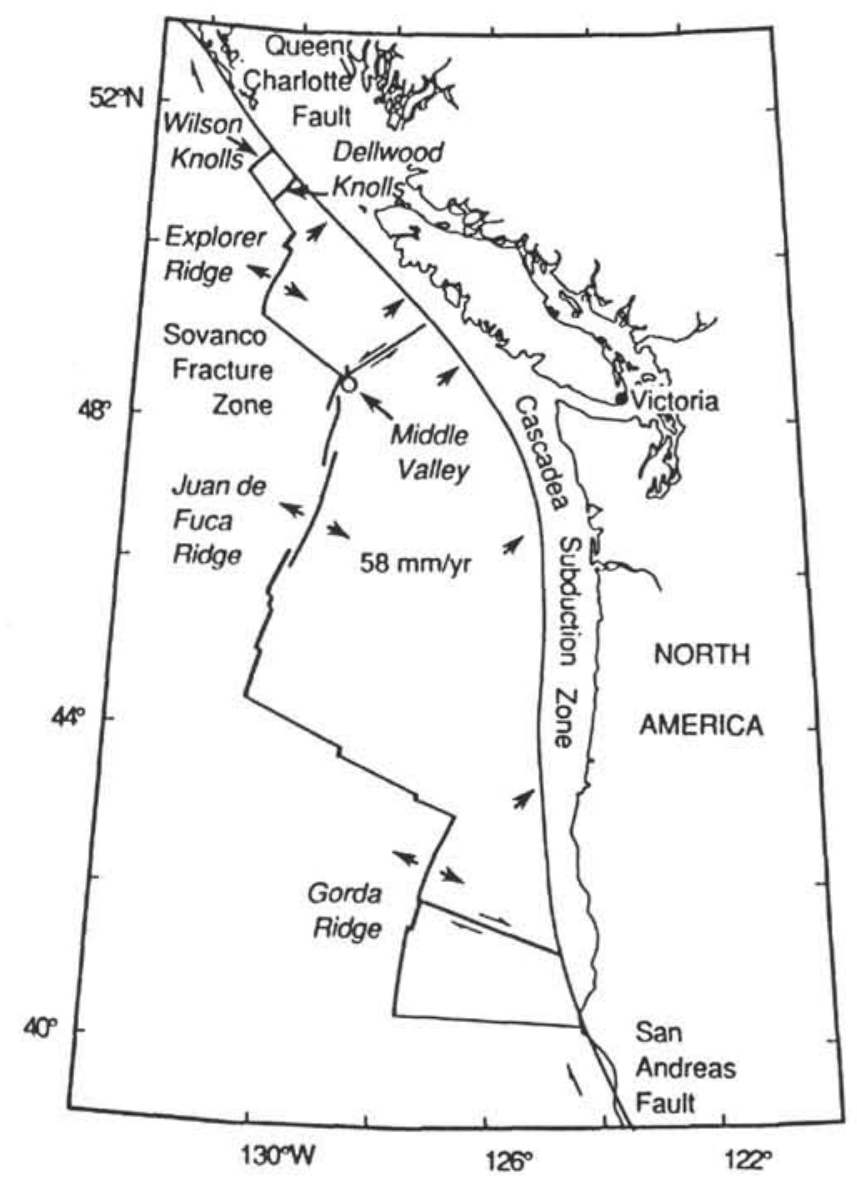

Figure 1. Location map of Middle Valley, northern Juan de Fuca Ridge.

below suggests that Hole $856 \mathrm{~A}$ (and probably Hole $856 \mathrm{~B}$ ) had much higher thermal gradients in the recent past.

Diagenetic or authigenic carbonates are present in various forms. Spherical or elliptical (elongate parallel to the bedding plane) nodules or incipient nodules (see Davis, Mottl, Fisher, et al., 1992, figs. 12 and 13 , p. 303), in which the carbonate cements preexisting sediments, are most common in Holes $856 \mathrm{~A}, 857 \mathrm{C}$, and $858 \mathrm{~A}$. The nodules have diameters up to $10 \mathrm{~cm}$. Occasionally, the carbonate nodules are concentrically zoned with darker rims and lighter interiors. In Holes $857 \mathrm{C}$ and $857 \mathrm{D}$ it is often difficult to distinguish between carbonatecemented beds that have been rounded by drilling and carbonate nodules that are simply carbonate-cemented sediments with a roughly spherical outer margin. Carbonate cements in these holes occur preferentially in the coarser-grained basal turbiditic sediments, whereas the overlying hemipelagic mudstones tend to be noncalcareous. Small, tubular nodules that appear to cement sediments that infilled burrow structures are the most common form of diagenetic carbonate in Hole 858D and also occur in Holes 858A and 858B. These nodules are usually 3 to $5 \mathrm{~cm}$ long and a few $\mathrm{mm}$ in diameter, and can be straight or branched. Small (a few $\mathrm{mm}$ in diameter), roughly spherical carbonate nodules are also common, particularly in Holes 858A, 858C, and 858D.

Several other less abundant forms of carbonate are present. Carbonate micronodules that have been identified only by X-radiography occur in some parts of Holes 858A (see Rigsby et al., this volume). These may be equivalent to the above-mentioned small nodules. Occasionally, larger carbonate nodules, embrittled by cementation, are broken and filled with pure carbonate veins (see Davis, Mottl, Fisher, et al., 1992, figs. 12 and 14, pp. 303-304). Unusual crab-shaped, calcitic growths are found in several sections of Hole 857C (see Davis, Mottl, Fisher, et al., 1992, fig. 15, p. 305). Single crystals of calcite spar, up to $1 \mathrm{~cm}$ on edge, occur in parts of Holes $858 \mathrm{~B}$ and $858 \mathrm{C}$. Disseminated,

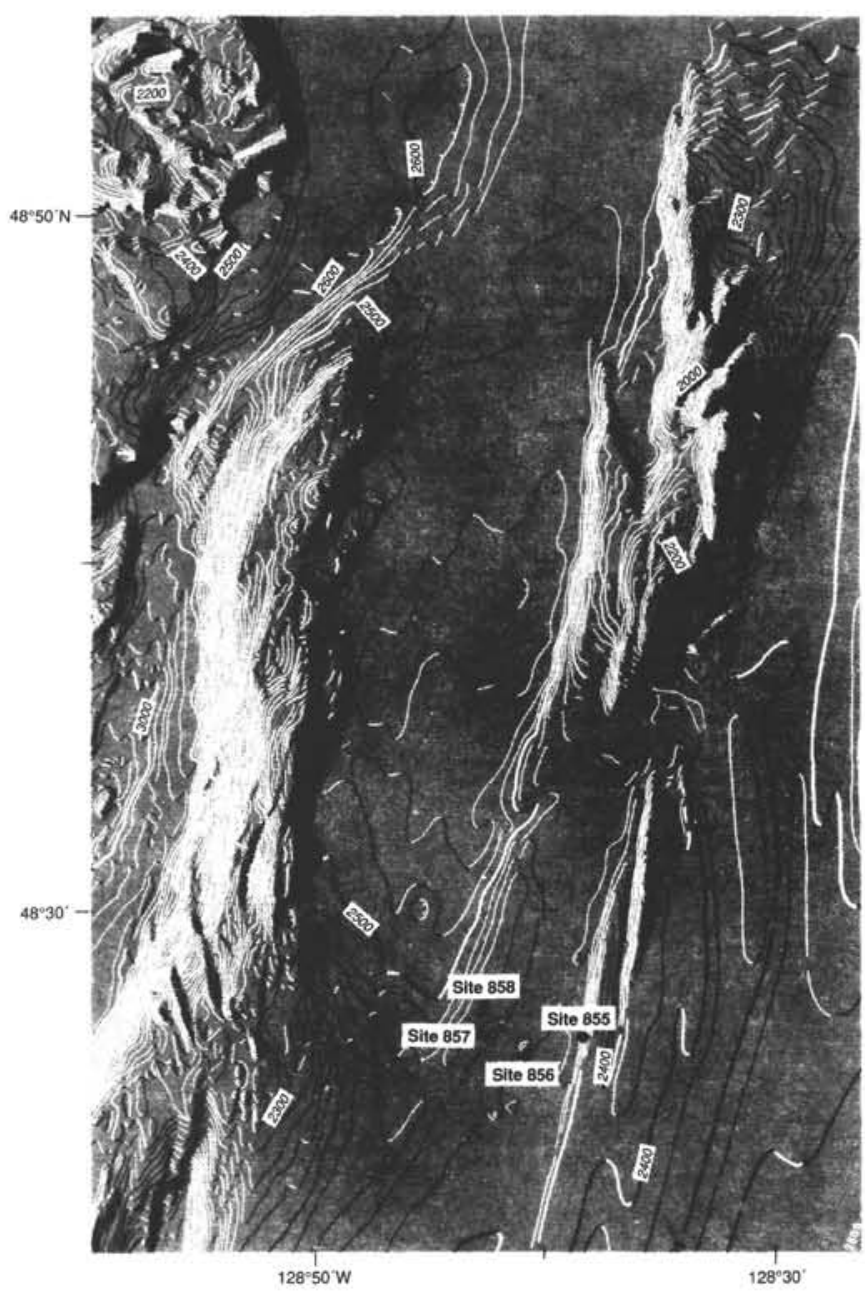

Figure 2. Site locations within Middle Valley.

zoned, euhedral dolomite crystals, about 50 micrometers on edge, occur in short intervals near the tops of Holes 858B and 858D. One limestone breccia, consisting of mud clasts cemented by calcite, occurs near the top of Hole 858C (see Davis, Mottl, Fisher, et al., 1992, fig. 15 , p. 450).

The shallowest occurrence of most of the diagenetic carbonate nodules and cements is near the base of the sediment that contains calcareous microfossils. In Hole 856A, the shallowest nodules identified occur at about $18 \mathrm{mbsf}$. Below $60 \mathrm{mbsf}$ in Hole $856 \mathrm{~A}$, the only carbonate present in the sediments occurs in the form of carbonate nodules. In Hole 856B, no carbonate nodules were reported and the carbonate content of sediments below $40 \mathrm{mbsf}$ is negligible. At Site 857 diagenetic carbonate occurs over an interval from about $46 \mathrm{mbsf}$ (in Hole $857 \mathrm{~A}$ ) to about $461 \mathrm{mbsf}$ (in Hole 857C). In Hole 858A carbonate nodules are restricted to depths between $11 \mathrm{mbsf}$ and $16 \mathrm{mbsf}$. In Hole $858 \mathrm{~B}$ carbonate nodules occur only between $3 \mathrm{mbsf}$ and about $5 \mathrm{mbsf}$. In Hole $858 \mathrm{C}$ carbonate nodules first appear at less than $1 \mathrm{mbsf}$, and no carbonate was observed below 50 mbsf. In Hole 858D, carbonate nodules occur at less than $1 \mathrm{mbsf}$, but were not reported below $23 \mathrm{mbsf}$.

The close coincidence at most sites of the depths of the zone of disappearance of calcareous microfossils and the zone of abundance of diagenetic carbonates suggests that diagenetic carbonate is partially derived from the dissolution of biogenic carbonate.

\section{METHODOLOGY}

Approximately 120 samples of carbonate cements and nodules were extracted from Holes $856 \mathrm{~A}, 857 \mathrm{C}, 858 \mathrm{~A}, 858 \mathrm{~B}, 858 \mathrm{C}$, and 
858D. The largest of the nodules were subsampled as many as five times each. Samples were dried and powdered. All samples were analyzed by powder X-ray diffraction (XRD) in order to determine the carbonate mineralogy and the approximate magnesium content of dolomites and calcites. Splits of these powders were also analyzed for strontium isotopic ratios, stable carbon and oxygen isotopic ratios, and major and minor elemental concentrations.

Strontium isotopic analyses were done on acetic acid leaches of sample powders. Strontium was isolated using a strontium-specific extraction resin (Horwitz et al., 1991). Samples were run on a VG Sector 54 mass spectrometer at the Department of Geology, University of North Carolina, Chapel Hill. Strontium isotopic ratios are normalized to ${ }^{86} \mathrm{Sr} /{ }^{88} \mathrm{Sr}=0.1194$. All ${ }^{87} \mathrm{Sr} /{ }^{86} \mathrm{Sr}$ ratios are relative to a value of 0.710250 for NBS SRM987. External precision is better than $\pm 2 \times 10^{-5}$.

Elemental concentrations were determined on buffered $(\mathrm{pH}=5.0)$ acetic acid-ammonium acetate leaches. Approximately $100 \mathrm{mg}$ of sample powder was dissolved for about $2 \mathrm{hr}$ in $10 \mathrm{ml}$ of acid. After centrifugation, the leachate was decanted and diluted for analysis. Elemental determinations were made by flame atomic absorption spectrophotometry (AAS) using a Perkin-Elmer 5000 AAS. Separate powder splits were subjected to the entire protocol in order to determine analytical precision. Mean relative errors are $0.46 \%$ for calcium, $1.29 \%$ for magnesium, $1.55 \%$ for strontium, $2.27 \%$ for iron, and $1.63 \%$ for manganese.

Carbon and oxygen stable isotopic ratios were analyzed on splits of the same sample powders. Approximately $10 \mathrm{mg}$ of sample was reacted in $100 \%$ phosphoric acid at $90^{\circ} \mathrm{C}$ (McCrea, 1950) in an online automated preparation system. The resulting $\mathrm{CO}_{2}$ was analyzed on a VG Prism II ratio mass spectrometer at the Geological Institute, University of Bern, Switzerland. Repeated analyses of standard materials yielded a reproducibility of better than 0.10 per mil $(\%)$ for oxygen and $0.05 \%$ for carbon. No correction was made for the difference in phosphoric acid fractionation factors between dolomite and calcite (i.e., all data were adjusted using the phosphoric acid fractionation factor for calcite at $90^{\circ} \mathrm{C}$ ). Values in Table 1 are reported relative to the PDB standard.

\section{RESULTS}

The results of the chemical, mineralogical, and isotopic analyses are given in Table 1. The most pertinent of these results are discussed in the following sections. It should be noted that all of the sediments and the carbonate nodules from Hole 858D, Core 4H (20.2 to 23.04 mbsf) are stratigraphically displaced as a result of coring artifacts. These sediments are all derived from higher in the section and may have fallen down the hole and been recored. For this reason, we consider variations of properties vs. depth for Cores 858D-1H and 858D$2 \mathrm{H}$ only, or we utilize the entire data set from Hole $858 \mathrm{D}$ by considering variations of properties using oxygen isotopic ratios of nodules as proxy indicator of depth.

\section{MINERALOGY AND MAGNESIUM CONTENT OF DIAGENETIC CARBONATES}

The diagenetic carbonates analyzed are composed of either lowmagnesium calcite (LMC), high-magnesium ( $>4$ molar percent [mol\%]) calcite (HMC), or dolomite (Table 1). Dolomites occur as nodules near the top of Hole $857 \mathrm{C}$, but are not found below $105 \mathrm{mbsf}$ in that hole. Dolomites also occur as disseminated individual rhombic crystals in a few intervals near the top of Hole 858D. These two occurrences are quite distinct and are discussed separately.

In both holes, the dolomites usually display superstructure reflections typical of well-ordered dolomites. In Hole $857 \mathrm{C}$ the dolomites contain 38.9 to $47.5 \mathrm{~mol} \%$ magnesium, whereas those in Hole 858D are, on average, significantly less magnesian.

In Hole $857 \mathrm{C}$, only one sample (857C-9R-1, 9-10 cm) contains both dolomite and calcite. In this case, the calcite is HMC, containing about $8 \mathrm{~mol} \%$ magnesium (determined by XRD) and the dolomite is a protodolomite which lacks superstructure reflections and contains about $40 \mathrm{~mol} \%$ magnesium (as determined by XRD). All of the carbonates at greater burial depths in Hole $857 \mathrm{C}$ are calcites. The magnesium content of these calcites generally decreases downward (Fig. 3).

The inferred paragenesis (i.e., downcore succession) of carbonate minerals at Hole $857 \mathrm{C}$ is an interesting one: ordered dolomite $\rightarrow$ protodolomite $\rightarrow \mathrm{HMC} \rightarrow \mathrm{LMC}$. The implied process, dedolomitization, is not uncommon in nature, but the intermediate phases have to our knowledge never been observed previously. The dedolomitization reaction can be written as:

$$
\mathrm{MgCa}\left(\mathrm{CO}_{3}\right)_{2}+\mathrm{Ca}^{2+}=2 \mathrm{CaCO}_{3}+\mathrm{Mg}^{2+},
$$

where the dissolved calcium in pore waters is probably supplied by anhydrite dissolution or plagioclase alteration. The magnesium/calcium molar ratio in present-day pore waters at the depth of apparent dedolomitization (110 mbsf) is about 0.5 (Davis, Mottl, Fisher, et al., 1992). At the approximate in-situ temperature of $80^{\circ} \mathrm{C}$ (Davis, Mottl, Fisher, et al., 1992), this ratio is still well inside the hypothesized stability field for dolomite according to Land (1987). The reason for this discrepancy is unknown at present.

In Hole 858D, dolomites occur as dispersed rhombs within sediments at depths of 12.3 to 13.6 mbsf. The dolomite rhombs often coexist with a small amount of HMC or LMC. Molar ratios of magnesium/calcium in pore waters in this depth range average about 0.28 (Davis, Mottl, Fisher, et al., 1992). These solutions are within the dolomite stability field (Baker, unpubl. data). At $200^{\circ} \mathrm{C}$ (approximately 20 mbsf), magnesium/calcium ratios in pore waters decrease to values as low as 0.08 . Experimental work has shown that dolomite should be unstable relative to calcite in the latter solutions (Rosenberg et al., 1967).

In sediments at depths shallower than 12.3 mbsf, HMC is authigenic. The magnesium content of the calcites decreases with increasing burial depth (Fig. 4). The shallowest carbonate, occurring at $0.46 \mathrm{mbsf}$, contains $11.3 \mathrm{~mol} \%$ magnesium. HMC of $11.3 \mathrm{~mol} \% \mathrm{Mg}$ is highly undersaturated in cold oceanic bottom waters at this water depth (2467 $\mathrm{m}$ ); thus it forms because of some process that maintains a high degree of supersaturation (relative to LMC or dolomite) of sedimentary pore fluids. This process, microbial sulfate reduction, is discussed in more detail in a following section.

A layer of aragonite was found in Hole $857 \mathrm{~A}-6 \mathrm{H}-4,65-66 \mathrm{~cm}$.

High pore water alkalinities were reported at this level (Davis, Mottl, Fisher, et al., 1992, p. 334).

\section{OXYGEN ISOTOPIC THERMOMETRY}

Oxygen isotopic compositions of diagenetic carbonates are plotted against sample depth for Hole 856A (Fig. 5), Hole 857C (Fig. 6), Hole 858A (Fig. 7), and Hole 858D, Cores $1 \mathrm{H}$ and $2 \mathrm{H}$ (Fig. 8). In the absence of reported measurements, we assume $\delta^{18} \mathrm{O}=0 \%$ (standard mean ocean water [SMOW]) for the oxygen isotopic composition of pore waters. Using the temperature dependence of the fractionation factor for oxygen isotopes between calcite and water (O'Neil et al., 1969), we can calculate temperatures of carbonate precipitation (Tc). Table 2 shows maximum calculated temperatures (minimum values of $\delta^{18} \mathrm{O}$ ) for each hole and compares those to present-day temperatures $\left(T_{m}\right)$ determined from in-situ measurements (Davis, Mottl, Fisher, et al., 1992). The generally good agreement between these two temperature determinations confirms the utility of isotopic geothermometry in these cores and is also evidence for the origin or rapid recrystallization of the carbonates at their present-day burial depths.

Information on present-day thermal gradients can be determined by examining the isotopic temperatures at Holes $857 \mathrm{C}$ and $858 \mathrm{D}$. The isotopic temperatures are shown for all of the calcites from Holes $857 \mathrm{C}$ (Fig. 9) and Cores 858D-1H and -2H (Fig. 10). Dolomite temperatures were calculated using a dolomite-calcite constant low-temperature fractionation of 3.1\%o (Fritz and Smith, 1970). At both sites, the increase of temperature with depth is nearly constant, about $10.5^{\circ} \mathrm{C} / \mathrm{m}$ at Hole 
Table 1. Geochemistry and mineralogy of diagenetic carbonates.

\begin{tabular}{|c|c|c|c|c|c|c|c|c|c|c|c|c|c|c|c|c|c|}
\hline $\begin{array}{l}\text { Core, section, } \\
\text { interval }(\mathrm{cm})\end{array}$ & Description & $\begin{array}{l}\text { Lab } \\
\text { sample \# }\end{array}$ & $\begin{array}{l}\text { Depth } \\
\text { (mbsf) }\end{array}$ & $\begin{array}{c}\delta^{13} \mathrm{C} \\
q_{\infty}, \mathrm{PDB}\end{array}$ & $\begin{array}{l}8^{18} \mathrm{O} \\
q_{\infty}, \text { PDB }\end{array}$ & Sr $87 / 86$ & $\begin{array}{l}\text { Mole\%Ca } \\
\text { dolomite }\end{array}$ & $\begin{array}{c}\text { Mole \%Ca } \\
\text { calcite }\end{array}$ & $\begin{array}{l}\text { Mole\%Mg } \\
\text { dolomitc }\end{array}$ & $\begin{array}{c}\text { Mole\% } \% \text { Mg } \\
\text { calcite }\end{array}$ & $\underset{\substack{\mathrm{Sr} / \mathrm{Ca} \\
(\mathrm{mol} / \mathrm{mol})}}{2}$ & $\begin{array}{c}\text { Mole \% Fe } \\
\text { calcite }\end{array}$ & $\begin{array}{l}\text { Mole\%Fe } \\
\text { dolonnite }\end{array}$ & $\begin{array}{c}\text { Mole\%Mn } \\
\text { calcite }\end{array}$ & $\begin{array}{c}\text { Mole\%Mn } \\
\text { dolomite }\end{array}$ & $\begin{array}{c}\mathrm{Zn} / \mathrm{Ca} \\
(\mathrm{mol} / \mathrm{mol})\end{array}$ & $\mathrm{X}$-ray \\
\hline \multicolumn{18}{|l|}{$139-856 \mathrm{~A}$ - } \\
\hline $3 \mathrm{H}-2,118-119$ & Small indurated nodule & 139310 & 14.53 & -10.65 & -8.51 & & & 88.67 & & 4.99 & $3.32 \mathrm{E}-04$ & 1.89 & & 4.45 & & 3.08E-05 & $\mathrm{H}$ \\
\hline $3 \mathrm{H}-7,34-35$ & Small indurated nodule & 139311 & 21.46 & -7.99 & -11.99 & & & 93.29 & & 3.88 & 3.64E-03 & 0.48 & & 2.35 & & $2.09 \mathrm{E}-05$ & L \\
\hline $6 \mathrm{H}-5,132-135$ & Indur. nodule, light gray zone & $139315-1$ & 48.02 & $-24,94$ & -9.27 & & & 94.26 & & 3.72 & $1.75 \mathrm{E}-04$ & 0.24 & & 1.77 & & 2.67E-05 & \\
\hline $\begin{array}{l}6 \mathrm{H}-5.132-135 \\
7 \mathrm{H}-6.80-85\end{array}$ & $\begin{array}{l}\text { Indur. nodule, dark gray crystalline } \\
\text { Large nodule }\end{array}$ & ${ }_{139319}^{139315-2}$ & $\begin{array}{l}48.02 \\
585\end{array}$ & -26.01 & $\begin{array}{r}-9.33 \\
-18.98\end{array}$ & & & 93.86 & & 4.09 & 1.83E-04 & 0.04 & & 2.01 & & $2.52 \mathrm{E}-05$ & $\mathrm{~L}$ \\
\hline $\begin{array}{l}7 \mathrm{H}-6.80-85 \\
8 \mathrm{H}-3,88-91\end{array}$ & $\begin{array}{l}\text { Large nodule } \\
\text { Large porly indur. nodule, outer }\end{array}$ & $\begin{array}{l}139319 \\
139320-1\end{array}$ & $\begin{array}{l}58.5 \\
63.58\end{array}$ & $\begin{array}{r}-17.34 \\
-17.18\end{array}$ & $\begin{array}{l}-18.98 \\
-12.97\end{array}$ & 0.705823 & & 95.94 & & 2.38 & $1.89 \mathrm{E}-04$ & 0.29 & & 1.39 & & $1.52 E-05$ & \\
\hline $8 \mathrm{H}-3,88-91$ & Large poorly indur. nodule, inner & $139320-2$ & 63.58 & -19.47 & -11.63 & 0.705823 & & 95.17 & & 3.06 & $1.84 \mathrm{E}-04$ & 0.39 & & 1.38 & & $1.61 \mathrm{E}-05$ & \\
\hline $8 \mathrm{H}-4,28-31$ & Large nodule, sandy & 139321 & 64.48 & -18.12 & & & & 96.97 & & 1.92 & $1.66 \mathrm{E}-04$ & 0.13 & & 0.98 & & $7.54 \mathrm{E}-06$ & $\mathrm{~L}$ \\
\hline $8 \mathrm{H}-4,102-106$ & Large nodule, sandy, gray outer zone & $139322-1$ & 65.22 & -18.33 & -12.21 & 0.705527 & & 97.37 & & 1.73 & $1.99 \mathrm{E}-04$ & 0.08 & & 0.81 & & 2.09E-05 & L \\
\hline $8 \mathrm{H}-4,102-106$ & Large nodule, sandy, dark gray inner zone & $139322-2$ & 65.22 & -25.92 & -9.82 & 0.705467 & & 93.20 & & 4.34 & $1.57 \mathrm{E}-04$ & 0.71 & & 1.75 & & $3.18 \mathrm{E}-05$ & \\
\hline $8 \mathrm{H}-\mathrm{CC}, 8-10$ & Small indurated nodules & 139323 & 69.49 & -19.64 & -10.65 & & & 92.75 & & 5.26 & $2.11 \mathrm{E}-04$ & 0.58 & & 1.42 & & $1.71 \mathrm{E}-03$ & $\mathrm{~L}$ \\
\hline $9 \mathrm{H}-7,23-25$ & Indur, nodule & 139324 & 78.43 & -5.77 & -12.61 & 0.705077 & & & & & & & & & & & $\mathrm{~L}$ \\
\hline $10 \mathrm{H}-1,92-94$ & Indur. nodule & 139326 & 79.62 & -14.97 & -11.27 & & & 92.81 & & 4.25 & 2.01E-04 & 1.17 & & 1.77 & & $1.80 \mathrm{E}-05$ & \\
\hline $10 \mathrm{H}-2,13-15$ & Indur. nodule, medium gray zone & $139327-1$ & 80.33 & -16.31 & -12.32 & & & 95.50 & & 1.90 & $1.88 \mathrm{E}-04$ & 0.26 & & 2.33 & & 1.37E-05 & \\
\hline $10 \mathrm{H}-2,13-15$ & Indur. nodule, dark gray zone & $139327-2$ & 80.33 & -16.28 & -10.92 & & & 91.11 & & 5.02 & $1.88 \mathrm{E}-04$ & 1.30 & & 2.57 & & $2.44 \mathrm{E}-05$ & \\
\hline \multicolumn{18}{|l|}{$139.857 \mathrm{C}-\mathrm{C}$} \\
\hline $\begin{array}{l}\text { 4R-CC, } 3-5 \\
4 \text { R-CC } 3-5\end{array}$ & $\begin{array}{l}\text { Large well-indur. nodule or sis, outer } \\
\text { arge well-indure nodule or sis middle }\end{array}$ & $139007-1$ & 76.1 & -14.23 & 0.26 & 0.708765 & 50.54 & & $\begin{array}{l}47.52 \\
46.55\end{array}$ & & $5.38 \mathrm{E}-04$ & & 1.41 & & 0.53 & $8.33 \mathrm{E}-05$ & $\mathrm{D}^{*}$ \\
\hline $\begin{array}{l}4 R-C C, 3-5 \\
4 R-C, 3-5\end{array}$ & $\begin{array}{l}\text { Large well-indur. nodule or siss middle } \\
\text { Large well-indur. nodule or sis, inner }\end{array}$ & $\begin{array}{l}1390007-2 \\
139007-3\end{array}$ & $\begin{array}{l}76.1 \\
76.1\end{array}$ & $\begin{array}{r}-12.27 \\
-15.62\end{array}$ & $\begin{array}{l}0.07 \\
0.41\end{array}$ & 0708711 & $\begin{array}{l}51.62 \\
52.57\end{array}$ & & $\begin{array}{l}46.55 \\
45.48\end{array}$ & & $\begin{array}{r}5.30 \mathrm{E}-04 \\
5.35 \mathrm{E}-04\end{array}$ & & 1.35 & & 0.48 & $\begin{array}{l}4.46 \mathrm{E}-05 \\
454 \mathrm{E} .05\end{array}$ & \\
\hline $6 \mathrm{R}-1,6 \rightarrow 7$ & $\begin{array}{l}\text { Large well-ndur., nodule or Sis, inner } \\
\text { Well-indur, sis or large nodule(?), outer }\end{array}$ & $139010-1$ & $\begin{array}{l}76.1 \\
86.3\end{array}$ & $\begin{array}{l}-15.62 \\
-16.11\end{array}$ & $\begin{array}{l}0.41 \\
-0.65\end{array}$ & 0.708684 & ${ }_{52.11}^{52.57}$ & & $\begin{array}{l}45.48 \\
40.49\end{array}$ & & $3.76 \mathrm{E}-04$ & & $\begin{array}{l}1.37 \\
5.68\end{array}$ & & $\begin{array}{l}0.58 \\
1.72\end{array}$ & $\begin{array}{l}4.54 \mathrm{E}-05 \\
9.81 \mathrm{E}-05\end{array}$ & $\mathrm{D}^{*}$ \\
\hline $6 \mathrm{R}-1,6-7$ & Well-indur. sis or large nodule(?), inner & $139010-3$ & 86.3 & -15.19 & -1.24 & 0.708672 & 54.34 & & 38.01 & & $3.29 \mathrm{E}-04$ & & 5.55 & & 2.10 & $6.65 \mathrm{E}-05$ & \\
\hline $7 \mathrm{R}-1,2-3$ & Large well-indur. nodule, outer & $139012-1$ & 95.2 & -16.02 & -1.84 & & 53.05 & & 40.19 & & 3.3IE-04 & & 4.74 & & 2.02 & 3.10E-05 & $\mathrm{D}^{*}$ \\
\hline $\begin{array}{l}7 \mathrm{R}-1,2-3 \\
7 \mathrm{R}-1,2-3\end{array}$ & $\begin{array}{l}\text { Large well-indur., nodule, inner } \\
\text { Large well-ininur nodule, inner }\end{array}$ & $\begin{array}{l}139012-2 \\
1390123\end{array}$ & 95.2 & $\begin{array}{r}-15.99 \\
-15.79\end{array}$ & $\begin{array}{r}-1.66 \\
-1.49\end{array}$ & & $\begin{array}{l}52,32 \\
5452\end{array}$ & & $\begin{array}{l}41.65 \\
3986\end{array}$ & & 3.20E-04 & & 4.43 & & 1.61 & $5.85 E-05$ & \\
\hline $\begin{array}{l}7 \mathrm{R}-1,2-3 \\
7 \mathrm{R}-1,2-3\end{array}$ & $\begin{array}{l}\text { Large well-indurt, nodulu,e, inner } \\
\text { Large well-indur, nodule, outer }\end{array}$ & $\begin{array}{l}139012-3 \\
139012-4\end{array}$ & 95.2 & $\begin{array}{r}-15.79 \\
-15.77\end{array}$ & $\begin{array}{l}-1.49 \\
-1.53\end{array}$ & & $\begin{array}{l}54.52 \\
52.77\end{array}$ & & $\begin{array}{l}39.86 \\
42.20\end{array}$ & & $\begin{array}{l}3.12 \mathrm{E}-04 \\
3.41 \mathrm{E}-04\end{array}$ & & $\begin{array}{l}4.24 \\
3.80\end{array}$ & & 1.38 & $\begin{array}{l}3.27 \mathrm{E}-05 \\
3.24 \mathrm{E}-05\end{array}$ & \\
\hline $\begin{array}{l}7 \mathrm{R}-1,1,2-3 \\
7 \mathrm{R}-1,2-3\end{array}$ & $\begin{array}{l}\text { Large well-indur, nodulue, outer } \\
\text { Large well-indur, nodule, outer }\end{array}$ & $\begin{array}{l}1390122-4 \\
139012-5\end{array}$ & $\begin{array}{l}95.2 \\
95.2\end{array}$ & $\begin{array}{l}-15.77 \\
-15.95\end{array}$ & $\begin{array}{l}-1.53 \\
-1.54\end{array}$ & & $\begin{array}{l}52.87 \\
52.81\end{array}$ & & $\begin{array}{l}\begin{array}{l}42.20 \\
41.82\end{array} \\
-\end{array}$ & & $\begin{array}{l}3.41 \mathrm{IE}-04 \\
3.26 \mathrm{E}-04\end{array}$ & & $\begin{array}{l}3.80 \\
4.01\end{array}$ & & $\begin{array}{l}1.23 \\
1.36\end{array}$ & $\begin{array}{l}3.24 \mathrm{E}-05 \\
4.84 \mathrm{E}-05\end{array}$ & \\
\hline $8 \mathrm{R}-\mathrm{CC}, 4-5$ & Large well- indur. nodule or sis/ms, outer & 139013-1 & 104.9 & -15.52 & -1.64 & & 53.27 & & 39.80 & & $3.78 \mathrm{E}-04$ & & 5.15 & & 1.78 & $6.92 \mathrm{E}-05$ & $\mathrm{D}^{*}$ \\
\hline 8R-CC, 45 & Large well-indur. nodule or sis/ms, middle & 139013-2 & 104.9 & -15.82 & -1.68 & & 54.80 & & 38.87 & & 3.61E-04 & & 4.69 & & 1.63 & 8.07E-05 & \\
\hline $\begin{array}{l}8 R-C C, 4-5 \\
8 R-C C, 4-5\end{array}$ & $\begin{array}{l}\text { Large well-indur. nodule or sis/ms, outer } \\
\text { arge }\end{array}$ & $\begin{array}{l}139013-3 \\
1390134\end{array}$ & 104.9 & -15.74 & -1.78 & & $\begin{array}{r}53.83 \\
53.89\end{array}$ & & 39.74 & & 3.69E-04 & & 4.72 & & 1.71 & $4.32 \mathrm{E}-05$ & \\
\hline $\begin{array}{l}8 R-C, 4-5 \\
9 R-1,9-10\end{array}$ & $\begin{array}{l}\text { Large well-indur. nodule or sis/ms, outer } \\
\text { Large well-indur. nodule or sis } / \mathrm{ms} \text {, outer }\end{array}$ & $\begin{array}{l}139013-4 \\
139014-1\end{array}$ & $\begin{array}{l}104.9 \\
114.6\end{array}$ & $\begin{array}{r}-15.75 \\
-18.09\end{array}$ & $\begin{array}{r}-1.77 \\
-561\end{array}$ & 0.708212 & 53.89 & 81.93 & 39.63 & 1281 & $\begin{array}{l}3.89 \mathrm{E}-04 \\
207 \mathrm{E}-04\end{array}$ & 1.25 & 4.77 & 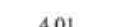 & 1.70 & $\begin{array}{l}7.11 \mathrm{E}-05 \\
\end{array}$ & HD* \\
\hline $9 R-1,9-10$ & $\begin{array}{l}\text { Large well-1ndur. noduce or sis/ms, outer } \\
\text { Large well-indur. nodule or sis/ms, inner }\end{array}$ & $\begin{array}{l}139014-2 \\
13901-1\end{array}$ & 114.6 & $\begin{array}{l}-18.09 \\
-17.83\end{array}$ & $\begin{array}{l}-3.01 \\
-3.54\end{array}$ & 0.708305 & & 81.93 & & 12.81 & $2.07 \mathrm{E}-04$ & 1.25 & & 4.01 & & $2.90 \mathrm{E}-05$ & $\mathrm{HD}^{*}$ \\
\hline $9 \mathrm{R}-1,9-10$ & Large well-indur, nodule or sis/ms, outer & 1390143 & 114.6 & -18.79 & -4.31 & 0.708393 & & 67.56 & & 26.29 & 3.63E-04 & 2.10 & & 4.05 & & 4.19E-05 & \\
\hline $\begin{array}{l}9 \mathrm{R}-1,9-10 \\
9 \mathrm{R}-1,49-50\end{array}$ & $\begin{array}{l}\text { Large well-indur., nodule or sis/ms, outer } \\
\text { Indurated sis/fine ss }\end{array}$ & $139014-\mathrm{A}$ & 114.6 & -17.1 & -7.01 & & & 87.29 & & 7.43 & $1.73 \mathrm{E}-04$ & 0.89 & & 4.38 & & 2.53E-05 & \\
\hline $\begin{array}{l}9 R-1,4-50 \\
9 R-1,49-50\end{array}$ & $\begin{array}{l}\text { Indurated sis/fine ss } \\
\text { Indurated sis/fine ss }\end{array}$ & $\begin{array}{l}139016-1 \\
190016-2\end{array}$ & $\begin{array}{l}115 \\
115\end{array}$ & $\begin{array}{r}-14.29 \\
-15.73\end{array}$ & $\begin{array}{l}-8.13 \\
-8.79\end{array}$ & 0.707428 & & & & 6.07 & & 1.53 & & 4.15 & & & \\
\hline $12 \mathrm{R}-1,8-9$ & $\begin{array}{l}\text { Indurated sisithine ss } \\
\text { Nodule, light gray zone }\end{array}$ & $139019-1$ & 143.6 & -17.39 & $\begin{array}{r}-8.79 \\
-10.09\end{array}$ & 0.70753 & & $\begin{array}{l}88.25 \\
91.07\end{array}$ & & $\begin{array}{l}6.07 \\
564\end{array}$ & $\begin{array}{l}1.82 \mathrm{E}-04 \\
2.24 \mathrm{E}-04\end{array}$ & $\begin{array}{l}1.53 \\
0.99\end{array}$ & & $\begin{array}{l}4.15 \\
2.30\end{array}$ & & $\begin{array}{l}2.44 \mathrm{E}-04 \\
606 \mathrm{E}-05\end{array}$ & ${ }_{H}^{\mathrm{H}}$. \\
\hline $12 \mathrm{R}-1.8-9$ & Nodule, dark gray zone & $139019-2$ & 143.6 & -17.48 & -10.01 & & & 91.53 & & 5.25 & $1.95 \mathrm{E}-04$ & 0.93 & & 2.28 & & $7.22 \mathrm{E}-05$ & $n$ \\
\hline $12 \mathrm{R}-1,47-48$ & Indurated fine ss/sis & 139021 & 144 & -16.06 & -11.18 & & & 95.22 & & 2.29 & $1.79 \mathrm{E}-04$ & 0.38 & & 2.11 & & $3.62 \mathrm{E}-05$ & $\mathrm{~L}$ \\
\hline${ }_{22 R-1,126-127}^{127}$ & Poorly indurated ms/ss & 139022 & 144.8 & -16.21 & -11.82 & & & 94.89 & & 2.95 & $1.91 \mathrm{E}-04$ & 0.37 & & 1.79 & & 2.34E-05 & L \\
\hline $\begin{array}{l}22 R-1,108-112 \\
22 R-1,108-112\end{array}$ & $\begin{array}{l}\text { IIdurated lamininated sis } \\
\text { Indurated laminated sis }\end{array}$ & $\begin{array}{l}139037-3 \\
139037-\mathrm{A}\end{array}$ & $\begin{array}{l}241.4 \\
241.4\end{array}$ & $\begin{array}{l}-11.87 \\
-12.05\end{array}$ & $\begin{array}{l}-13.15 \\
-13.62\end{array}$ & 07069 & & 94.18 & & 404 & $166 \mathrm{E}_{-04}$ & 104 & & 075 & & & $\mathrm{~h}$ \\
\hline $22 \mathrm{R}-1,108-112$ & $\begin{array}{l}\text { Indurated laminataced sis } \\
\text { Indurated laminated sis }\end{array}$ & $139037-\mathrm{B}$ & 241.4 & $\begin{array}{l}-12.05 \\
-11.89\end{array}$ & $\begin{array}{l}-13.62 \\
-13.48\end{array}$ & 0.7069 & & 94.18 & & & $1.06 \mathrm{E}-04$ & 1.04 & & & & & $L^{-}$ \\
\hline $22 \mathrm{R}-3,1-5$ & Large nodule, color-zoned; innermost zone & $139038-1$ & 242 & -10.89 & -14.6 & 0.70701 & & & & & & & & & & & \\
\hline $\begin{array}{l}22 R-3,1-5 \\
22 R-3,-5\end{array}$ & Large nodule, color-zoned; 2nd zone & 139038-2 & 242 & -8.9 & $-14,49$ & 0.706754 & & & & & & & & & & & \\
\hline $\begin{array}{l}222-3,-3,-5 \\
22 R-3,1-5\end{array}$ & $\begin{array}{l}\text { Large nodule, color-zoned; ; } 3 \text { rd zone } \\
\text { Large nodule, color-zoned outermost zone }\end{array}$ & $\begin{array}{l}139938-3 \\
139038.4\end{array}$ & ${ }_{242}^{242}$ & $\begin{array}{l}-7.46 \\
-6.61\end{array}$ & $\begin{array}{r}-15.23 \\
-11.21\end{array}$ & $\begin{array}{l}0.706905 \\
0.706298\end{array}$ & & & & & & & & & & & \\
\hline $24 \mathrm{R}-1,31-35$ & Indurated laminated sis/ms; light gray band & $139039-3$ & 259.5 & -11.14 & -14.57 & 0.700298 & & 96.47 & & 2.13 & $2.27 \mathrm{E}-04$ & 0.55 & & 0.85 & & 2.38E-05 & L. \\
\hline $25 \mathrm{R}-1,41-43$ & Indur. sis/ms; light-colored lense, inner & $139041-2$ & 269.3 & -7.74 & -14.99 & & & 95.47 & & 1.88 & $1.83 \mathrm{E}-04$ & 0.55 & & 2.09 & & $2.12 \mathrm{E}-05$ & \\
\hline $25 \mathrm{R}-1,41-43$ & Indur. sis/ms; light-colored lense, outer & $139041-\mathrm{A}$ & 269 & -8.24 & -14.82 & & & 95.39 & & 2.06 & $1.85 \mathrm{E}-04$ & 0.57 & & 1.99 & & 2.03E-05 & L \\
\hline 26R-CC, $1-3$ & Large thin nodule, zoned; light gray zone, outer & 139043-1 & 276.2 & -14.5 & -15.88 & & & 94.19 & & 3.73 & 2.21E-04 & 1.21 & & & & 4.87E-05 & $\mathrm{L}^{*}$ \\
\hline $\begin{array}{l}26 \mathrm{R}-\mathrm{CC}, 1-3 \\
26 \mathrm{R}-\mathrm{CC}, 1-3\end{array}$ & Large thin nodule, zoned, light gray zone, inner & $139043-2$ & 276.2 & -15.26 & -16.85 & & & $\begin{array}{l}94.56 \\
93.60\end{array}$ & & 3.38 & 2.69E-04 & 1.07 & & 0.99 & & 4.11E-05 & \\
\hline $\begin{array}{l}26 \mathrm{R}-\mathrm{CC}, 1-3 \\
26 \mathrm{R}-\mathrm{CC}, 1-3\end{array}$ & $\begin{array}{l}\text { Large thin nodule, zoncd; light gray zone, outer } \\
\text { Large thin }\end{array}$ & 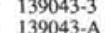 & $\begin{array}{l}276.2 \\
2762\end{array}$ & $\begin{array}{l}-14.12 \\
-14.74\end{array}$ & $\begin{array}{l}-16.32 \\
-1651\end{array}$ & 0705913 & & $\begin{array}{l}93.60 \\
94.81\end{array}$ & & $\begin{array}{l}4.12 \\
3.21\end{array}$ & $\begin{array}{l}2.08 \mathrm{E}-04 \\
2.43 \mathrm{E}-04\end{array}$ & 1.44 & & $\begin{array}{l}0.85 \\
0.88\end{array}$ & & $\begin{array}{l}7.98 \mathrm{E}-05 \\
3.895\end{array}$ & \\
\hline $26 \mathrm{R}-\mathrm{CC}, 1-3$ & $\begin{array}{l}\text { Large thin nowine, zoned; darker outer zone } \\
\text { Large the zoned }\end{array}$ & 139043-B & 276.2 & $\begin{array}{l}-14.74 \\
-10.33\end{array}$ & $\begin{array}{l}-16.31 \\
-14.65\end{array}$ & 0.706981 & & 94.27 & & 3.22 & $\begin{array}{l}2.435 \mathrm{E}-04 \\
1.74\end{array}$ & 1.11 & & $\begin{array}{l}0.88 \\
1.40\end{array}$ & & $\begin{array}{l}3.89 \mathrm{E}-03 \\
1.2 \mathrm{E}-04\end{array}$ & \\
\hline
\end{tabular}


Table 1 (continued).

\begin{tabular}{|c|c|c|c|c|c|c|c|c|c|c|c|c|c|c|c|c|c|}
\hline $\begin{array}{l}\text { Core, section, } \\
\text { interval (cm) }\end{array}$ & Description & $\begin{array}{l}\text { Lab } \\
\text { sample \# }\end{array}$ & $\begin{array}{l}\text { Depth } \\
\text { (mbsf) }\end{array}$ & $\begin{array}{c}\delta_{0}^{13} \mathrm{C} \\
\psi_{0}, \mathrm{PDB}\end{array}$ & $\begin{array}{c}\delta^{18} \mathrm{O} \\
\psi_{\infty}, \mathrm{PDB}\end{array}$ & Sr $87 / 86$ & $\begin{array}{l}\text { Mole\%Ca } \\
\text { dolomite }\end{array}$ & $\begin{array}{c}\text { Mole \%Ca } \\
\text { calcite }\end{array}$ & $\begin{array}{c}\text { Mole\% Mg } \\
\text { dolomite }\end{array}$ & $\begin{array}{c}\text { Mole\% } \% \mathrm{Mg} \\
\text { calcite }\end{array}$ & $\begin{array}{c}\mathrm{Sr} / \mathrm{Ca} \\
(\mathrm{mol} / \mathrm{mol})\end{array}$ & $\begin{array}{c}\text { Mole\%Fe } \\
\text { calcite }\end{array}$ & $\begin{array}{l}\text { Mole\%Fe } \\
\text { dolomite }\end{array}$ & $\begin{array}{c}\text { Mole\%Mn } \\
\text { calcite }\end{array}$ & $\begin{array}{c}\text { Mole\%Mn } \\
\text { dolomite }\end{array}$ & $\begin{array}{c}\mathrm{Zn} / \mathrm{Ca} \\
(\mathrm{mol} / \mathrm{mol})\end{array}$ & $\mathrm{X}$-ray \\
\hline $36 \mathrm{R}-2,35-37$ & Well-indurated ms & 139067-2 & 347.9 & -13.69 & -19.51 & & & 96.52 & & 1.94 & $3.34 \mathrm{E}-04$ & 0.58 & & 0.96 & & $1.85 \mathrm{E}-05$ & $\mathrm{~L}$ \\
\hline $36 \mathrm{R}-2,61-63$ & Well-indurated ms & $139068-2$ & 348.2 & -13.14 & -19.28 & & & 95.99 & & 2.24 & $3.59 \mathrm{E}-04$ & 0.77 & & 1.00 & & $2.06 \mathrm{E}-05$ & $\mathrm{~L}$ \\
\hline $37 \mathrm{R}-1,104-106$ & Large nodule, sand-size grains & 139071 & 351.8 & -11.27 & -18.31 & 0.706482 & & 95.76 & & 2.38 & $4.90 \mathrm{E}-04$ & 0.65 & & 1.21 & & $7.15 \mathrm{E}-05$ & $\mathrm{~L}$ \\
\hline $39 \mathrm{R}-1,57-59$ & Nodule(?) & $139075-1$ & 361 & -17.18 & -18.77 & & & 94.05 & & 4.69 & $2.85 \mathrm{E}-04$ & 0.57 & & 0.69 & & $1.44 \mathrm{E}-05$ & $\mathrm{~L}$ \\
\hline $39 \mathrm{R}-1,57-59$ & Nodule(?); dark crystalline mat'I & $139075-2$ & 361 & $\begin{array}{c}-16.59 \\
1589\end{array}$ & -16.74 & & & 95.79 & & 2.67 & $2.18 \mathrm{E}-04$ & 0.41 & & 1.13 & & & $\mathrm{~L}$ \\
\hline $\begin{array}{l}39 R-2,107-109 \\
4 R-1,128-130\end{array}$ & Indur. sis/fine ss w/dark (organic?) layer & 139077 & 363 & -15.81 & -19.19 & & & 95.86 & & 3.38 & $2.74 \mathrm{E}-04$ & 0.35 & & 0.40 & & $1.66 \mathrm{E}-05$ & $\mathrm{~L}$ \\
\hline $\begin{array}{l}458-1,128-130 \\
48 R-1,40-42\end{array}$ & $\begin{array}{l}\text { Indur. ms/sis w/light-colored lense shaped layer } \\
\text { Well-ininur ms }\end{array}$ & $\begin{array}{l}139093-2 \\
13909-1\end{array}$ & 305.6 & $\begin{array}{r}-13.09 \\
-9.68\end{array}$ & $\begin{array}{l}-21.63 \\
-21.78\end{array}$ & 0.705894 & & $\begin{array}{r}97.50 \\
05.53\end{array}-1010$ & & $\begin{array}{l}1.38 \\
2.46\end{array}$ & $\begin{array}{l}1.74 \mathrm{E}-04 \\
209 \mathrm{E}-04\end{array}$ & 0.42 & & 0.70 & & $\begin{array}{l}2.70 \mathrm{E}-05 \\
505 \mathrm{~F}-05\end{array}$ & $L$ \\
\hline $50 R-1,102-105$ & & $\begin{array}{l}139098-1 \\
139104-1\end{array}$ & 419.3 & -11.86 & $\begin{array}{l}-21.5 \\
-21.5\end{array}$ & 0.706072 & & $\begin{array}{l}95.53 \\
96.02\end{array}$ & & $\begin{array}{l}2.46 \\
2.23\end{array}$ & $\begin{array}{l}2.09 \mathrm{E}-04 \\
1.59 \mathrm{E}-04\end{array}$ & $\begin{array}{l}0.65 \\
0.64\end{array}$ & & $\begin{array}{l}1.37 \\
1.11\end{array}$ & & $\begin{array}{l}5.05 E-05 \\
1.75 E-05\end{array}$ & ${ }_{\mathrm{L}}^{\mathrm{L}}$ \\
\hline $50 \mathrm{R}-1,102-105$ & Well-indur. ms, It. gray cemented zone & $139104-2$ & 419.3 & $-10,42$ & -21.69 & 0.706108 & & & & & & & & & & & minor $\mathrm{L}$ \\
\hline \multicolumn{18}{|l|}{ 139-858A- } \\
\hline $2 \mathrm{H}-6,99-100$ & Small nodule w/clam shell & 139138 & 10.9 & -26.85 & 2.71 & & & 96.60 & & 3.02 & $3.60 \mathrm{E}-04$ & 0.19 & & 0.19 & & $2.82 \mathrm{E}-05$ & $\mathrm{~L}$ \\
\hline $2 \mathrm{H}-7,28-30$ & Small nodule & 139139 & 11.68 & -35.41 & 3.03 & & & 96.40 & & 3.13 & $2.59 \mathrm{E}-04$ & 0.13 & & 0.33 & & $1.93 \mathrm{E}-05$ & L \\
\hline $\begin{array}{l}2 \mathrm{H}-7,30-31 \\
2 \mathrm{H}-7,30-31\end{array}$ & Small nodule, interior & $139140-1$ & 11.7 & -25.45 & 2.56 & & & 95.08 & & 3.59 & $3.92 \mathrm{E}-04$ & 0.11 & & 1.22 & & 4.11E-05 & $\mathrm{L}$ \\
\hline $\begin{array}{l}2 \mathrm{H}-7,30-31 \\
2 \mathrm{H}-7,32-33\end{array}$ & $\begin{array}{l}\text { Small nodule, margin } \\
\text { Small nocule, inner }\end{array}$ & $\begin{array}{l}139140-2 \\
19314-1\end{array}$ & $\begin{array}{l}11.7 \\
11.72\end{array}$ & $\begin{array}{r}-26.53 \\
-35.46\end{array}$ & $\begin{array}{l}3.74 \\
3.36\end{array}$ & 0.705974 & & 94.34 & & 4.63 & $4.78 \mathrm{E}-04$ & 0.12 & & 0.92 & & $2.57 \mathrm{E}-05$ & $\mathrm{~L}$ \\
\hline $2 \mathrm{H}-7,32-33$ & $\begin{array}{l}\text { Smail nodulue, inner } \\
\text { Small nodule, middle }\end{array}$ & $\begin{array}{l}139141-1 \\
139141-2\end{array}$ & $\begin{array}{l}11.72 \\
11.72\end{array}$ & 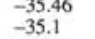 & $\begin{array}{l}3.36 \\
2.71\end{array}$ & & & & & & & & & & & & ${ }_{\mathrm{L}}^{\mathrm{L}}$ \\
\hline $2 \mathrm{H}-7,32-33$ & Small nodule, outer & $139141-3$ & 11.72 & -34.89 & 3.24 & & & 96.33 & & 3.12 & 2.33E-04 & 0.21 & & 0.34 & & $2.44 \mathrm{E}-05$ & $\mathrm{~L}$ \\
\hline $3 \mathrm{H}-1,66-68$ & Small nodule & 139142 & 12.6 & -36.86 & 3.84 & & & 95.77 & & 3.58 & $5.10 \mathrm{E}-04$ & 0.03 & & 0.62 & & $1.97 \mathrm{E}-05$ & L \\
\hline $\begin{array}{l}5 \mathrm{H}-2,2-4 \\
5 \mathrm{H}-2,24\end{array}$ & Nodule, zoned; inner zone & $\begin{array}{l}139159-1 \\
1301502\end{array}$ & 32.4 & -9.22 & -10.06 & 0706734 & & & & & & & & & & & $\mathrm{~L}$ \\
\hline $\begin{array}{l}5 \mathrm{H}-2,2,4 \\
5 \mathrm{H}-4,63-65\end{array}$ & $\begin{array}{l}\text { Nodule, zoned; outer zone } \\
\text { Small nodules }\end{array}$ & $\begin{array}{l}139159-2 \\
139164\end{array}$ & $\begin{array}{l}32.4 \\
36\end{array}$ & $\begin{array}{l}-10.14 \\
-18.55\end{array}$ & $\begin{array}{l}-10.95 \\
-13.89\end{array}$ & $\begin{array}{l}0.706734 \\
0.707359\end{array}$ & & 94.29 & & 4.14 & 2.94E-04 & 0.43 & & 114 & & $3875=05$ & \\
\hline $5 \mathrm{H}-6,40-45$ & $\begin{array}{l}\text { Small nodules } \\
\text { Notule }\end{array}$ & $\begin{array}{l}139164 \\
139167\end{array}$ & 38.8 & -13.31 & -11.48 & & & 94.18 & & 3.16 & $1.87 \mathrm{E}-04$ & 0.76 & & 1.90 & & $\begin{array}{l}3.87 \mathrm{E}-05 \\
1.99 \mathrm{E}-05\end{array}$ & L \\
\hline $5 \mathrm{H}-\mathrm{CC}, 10-11$ & Nodule, zoned; inner & $139169-1$ & 40 & -12.32 & -10.79 & & & 93.95 & & 2.90 & $2.03 \mathrm{E}-04$ & 0.93 & & 2.22 & & $\begin{array}{l}1.99 \mathrm{E}-05 \\
1.93-05\end{array}$ & ${ }_{\mathrm{L}}^{\mathrm{L}}$ \\
\hline $5 \mathrm{H}-\mathrm{CC}, 10-11$ & Nodule, zoned; outer & $139169-2$ & 40 & -8.12 & -13.95 & & & & & & & & & & & & $\mathrm{~L}$ \\
\hline $\begin{array}{l}6 \mathrm{H}-3,61-63 \\
9 \mathrm{X}-1,25-28\end{array}$ & $\begin{array}{l}\text { Nodule } \\
\text { Poorly indurated ms or nodule }\end{array}$ & 139172 & 44 & $\begin{array}{r}-16.74 \\
-1608\end{array}$ & -13.87 & & & 94.16 & & 3.69 & $2.20 \mathrm{E}-04$ & 1.10 & & 1.06 & & 4.91E-05 & \\
\hline 12X-CC, 13-14 & $\begin{array}{l}\text { Poorly indurated ms or nodule } \\
\text { Large nodule or well-indur. ms }\end{array}$ & $\begin{array}{l}139980 \\
139187\end{array}$ & $\begin{array}{l}62.7 \\
81.9\end{array}$ & $\begin{array}{l}-16.98 \\
-15.45\end{array}$ & $\begin{array}{l}-11.97 \\
-18.73\end{array}$ & & & ${ }_{92.41}^{94.21}$ & & $\begin{array}{l}3.07 \\
6.25\end{array}$ & $\begin{array}{l}1.62 \mathrm{E}-04 \\
3.67 \mathrm{E}-04\end{array}$ & $\begin{array}{l}0.81 \\
0.63\end{array}$ & & $\begin{array}{l}1.91 \\
0.71\end{array}$ & & $\begin{array}{l}2.82 \mathrm{E}-05 \\
2.10 \mathrm{E}-05\end{array}$ & \\
\hline \multicolumn{18}{|l|}{ 139-858B. } \\
\hline $2 \mathrm{H}-2,24-25$ & Silty mud/muddy silt & 139298 & 8.94 & -17.49 & -6.1 & & 36.01 & & 63.99 & & $1.98 \mathrm{E}-03$ & & & & & $2.36 \mathrm{E}-04$ & minor $\mathrm{D}$ \\
\hline $2 \mathrm{H}-2,26-27$ & Silty mud/muddy silt & 139299 & 8.96 & -12.84 & -8.37 & & 44.67 & & 55.18 & & $1.03 \mathrm{E}-03$ & & & & 0.16 & $9.63 \mathrm{E}-05$ & D \\
\hline $2 \mathrm{H}-2,29-30$ & Silty mud/muddy silt & 139300 & 8.99 & $\begin{array}{l}-12.87 \\
-12.87\end{array}$ & -8.17 & & 47.28 & & 52.59 & & $8.10 \mathrm{E}-04$ & & & & 0.13 & $1.75 \mathrm{E}-04$ & D \\
\hline \multirow{2}{*}{\multicolumn{18}{|c|}{$\begin{array}{l}139-858 \mathrm{C}- \\
2 \mathrm{H}-6.50-52\end{array}$}} \\
\hline $2 \mathrm{H}-6,50-52$ & Mud & 139215 & 12.8 & -25.08 & -14.01 & & & & & & & & & & & & \\
\hline $2 \mathrm{H}-7,33-35$ & Small nodulefindur. ms "lump": inner & $139216-1$ & & & & & & 95.23 & & 3.22 & $1.57 \mathrm{E}-04$ & 0.21 & & 1.34 & & $2.69 \mathrm{E}-05$ & $\mathrm{~L}^{*}$ \\
\hline $\begin{array}{l}2 \mathrm{H}-7,33-35 \\
14 \mathrm{X}-\mathrm{CC}, 8-9\end{array}$ & $\begin{array}{l}\text { Small nodulefindur. ms "lump"; outer } \\
\text { Small nodules? }\end{array}$ & $\begin{array}{l}139216-2 \\
139224\end{array}$ & 84.3 & -16.52 & -13.09 & & & 93.55 & & 4.96 & $2.29 \mathrm{E}-04$ & $\begin{array}{l}0.19 \\
4.38\end{array}$ & & 1.31 & & $5.54 \mathrm{E}-05$ & 1 \\
\hline \multicolumn{18}{|l|}{ 139-858D- } \\
\hline $1 \mathrm{H}-1,46-48$ & Poorly indur. clump/nodule & 139226 & 0.46 & -35.85 & 3.06 & 0.706216 & & 88.61 & & 11.33 & $5.23 \mathrm{E}-04$ & 0.06 & & 0.01 & & 1.75E-05 & $\mathrm{H}$ \\
\hline $1 \mathrm{H}-1,51-55$ & Poorly indur, clump/nodule & 139227 & 0.51 & -33.56 & 2.86 & & & 90.95 & & 8.87 & $4,12 \mathrm{E}-04$ & 0.03 & & 0.15 & & $1.63 \mathrm{E}-05$ & $\ddot{H}$ \\
\hline $1 \mathrm{H}-1,92-94$ & Nodule & 139228 & 0.92 & -27.52 & 2.67 & 0.706143 & & 94.19 & & 5.06 & $3.51 \mathrm{E}-04$ & 0.03 & & 0.73 & & $1.49 \mathrm{E}-05$ & $\mathrm{H}$ \\
\hline$-1,114-117$ & & 139229 & 1.1 & -31.94 & 2.06 & & & 92.01 & & 7.62 & $3.84 \mathrm{E}-04$ & 0.04 & & 0.33 & & $2.72 \mathrm{E}-05$ & ${ }_{\mathrm{H}}^{\mathrm{n}}$ \\
\hline $1 \mathrm{H}-2,57-59$ & Poorly indur. clump/nodule & 139231 & 2.1 & -26.08 & 1.14 & 0.705564 & & 90.70 & & 7.24 & $2.47 \mathrm{E}-04$ & 1.06 & & 1.00 & & $2.00 \mathrm{E}-05$ & $\mathrm{H}$ \\
\hline $1 \mathrm{H}-3,132-135$ & Nodule & 1392 & 4.3 & -25.25 & -2.21 & 0.705451 & & 91.94 & & 5.13 & $1.74 \mathrm{E}-04$ & 1.76 & & 1.16 & & $1.91 \mathrm{E}-05$ & $\mathrm{H}$ \\
\hline $1 \mathrm{H}-4,89-91$ & Small nodule & 139236 & 5.4 & -16.97 & -7.28 & 0.707414 & & 86.33 & & 8.82 & $4.20 \mathrm{E}-04$ & 3.05 & & 1.80 & & $3.14 \mathrm{E}-05$ & $\mathrm{H}$ \\
\hline 1H-5, 13-15 & Nodule (burrow-filling?) & 139237 & 6.13 & $\begin{array}{r}-17.54 \\
-16.40\end{array}$ & -8.65 & & & 91.21 & & $\begin{array}{l}4.74 \\
500\end{array}$ & $2.81 E-04$ & 2,79 & & 1.26 & & 3.34E-05 & $\mathrm{H}$ \\
\hline $\begin{array}{l}\text { 1H-5, 56-58 } \\
1 \mathrm{H}-5,64-67\end{array}$ & $\begin{array}{l}\text { Nodule } \\
\text { Nodule (burrow-filling) }\end{array}$ & $\begin{array}{l}139239 \\
139240\end{array}$ & $\begin{array}{l}6.56 \\
6.64\end{array}$ & $\begin{array}{r}-16.49 \\
-18.29\end{array}$ & $\begin{array}{l}-8.77 \\
-9.96\end{array}$ & & & 91.37 & & 5.00 & $3.52 \mathrm{E}-04$ & 2.16 & & 1.47 & & 3.36E-05 & $\underset{\mathrm{H}}{\mathrm{H}}$ \\
\hline $1 \mathrm{H}-5,76-80$ & Nodule (burrow-filling) & 139241 & 6.76 & $\begin{array}{l}-10.29 \\
-18.07\end{array}$ & -9.83 & & & 91.42 & & 4.90 & $2.72 \mathrm{E}-04$ & 2.25 & & 1.43 & & 2.43E-05 & $\mathrm{H}$ \\
\hline 1H-5, 89-93 & Nodule (burrow-filling) & & 6.89 & -18.25 & -10.22 & & & 91.68 & & 4.65 & $2.91 \mathrm{E}-04$ & 2. & & 1.44 & & $2.68 \mathrm{E}-05$ & $\mathrm{H}$ \\
\hline IH- $6.42-45$ & Small nodules & 1392 & 79 & -18.2 & -11.06 & & & 92.59 & & 4.72 & $2,17 \mathrm{E}-04$ & 1.82 & & 0.87 & & $3.28 \mathrm{E}-05$ & $\mathrm{H}$ \\
\hline IH-6, 57-59 & Small nodules & 139246 & 8.1 & -16.6 & -11.2 & & & 93.98 & & 2.89 & $2.40 \mathrm{E}-04$ & 2.46 & & 0.67 & & $3.78 \mathrm{E}-05$ & $\mathrm{H}$ \\
\hline 1H-6,90-93 & $\begin{array}{l}\text { Very small nodules } \\
\text { Verr small nodules }\end{array}$ & $\begin{array}{l}139247 \\
139248\end{array}$ & 8.4 & $\begin{array}{l}-17.03 \\
-14.02\end{array}$ & $\begin{array}{l}-12.35 \\
-11.4\end{array}$ & 0.704631 & & $\begin{array}{l}92.58 \\
90.24\end{array}-10$ & & $\begin{array}{l}5.05 \\
6.17\end{array}$ & $\begin{array}{l}3.88 \mathrm{E}-04 \\
229 \mathrm{-}-04\end{array}$ & $\begin{array}{l}1.69 \\
2.86\end{array}$ & & $\begin{array}{l}0.69 \\
0.72\end{array}$ & & $\begin{array}{l}4.48 \mathrm{E}-05 \\
1.68 \mathrm{E}-04\end{array}$ & $\underset{\mathrm{HD}}{\mathrm{H}}$ \\
\hline $\begin{array}{l}1 \mathrm{H}-\mathrm{CC}, 8-10 \\
2 \mathrm{H}-2,62-64\end{array}$ & $\begin{array}{l}\text { Very smaaln nodules } \\
\text { Mud clumps/sm nodules(?) }\end{array}$ & $\begin{array}{l}1399248 \\
139252\end{array}$ & $\begin{array}{r}9.1 \\
11.4\end{array}$ & $\begin{array}{l}-14.02 \\
-14.13\end{array}$ & $\begin{array}{l}-11.4 \\
-14.98\end{array}$ & & & $\begin{array}{l}90.244 \\
95.83\end{array}$ & & $\begin{array}{l}6.17 \\
2.62\end{array}$ & $\begin{array}{l}2.29 \mathrm{E}-04 \\
1.53 \mathrm{E}-04\end{array}$ & $\begin{array}{l}2.86 \\
0.62\end{array}$ & & $\begin{array}{l}0.72 \\
0.93\end{array}$ & & $\begin{array}{l}1.68 \mathrm{E}-04 \\
2.37-05\end{array}$ & $\underset{\mathrm{L}}{\mathrm{HD}}$ \\
\hline
\end{tabular}


Table 1 (continued).

\begin{tabular}{|c|c|c|c|c|c|c|c|c|c|c|c|c|c|c|c|c|c|}
\hline $\begin{array}{l}\text { Core, section, } \\
\text { interval }(\mathrm{cm})\end{array}$ & Description & $\begin{array}{c}\text { Lab } \\
\text { sample\# }\end{array}$ & $\begin{array}{l}\text { Depth } \\
\text { (mbst) }\end{array}$ & $\begin{array}{c}8^{13} \mathrm{C} \\
x_{0}, \mathrm{PDB}\end{array}$ & $\begin{array}{c}\delta_{0}^{18} \mathrm{O} \\
x_{0}, \mathrm{PDB}\end{array}$ & Sr $87 / 86$ & $\begin{array}{l}\text { Mole\%Ca } \\
\text { dolomite }\end{array}$ & $\begin{array}{l}\text { Mole \%ca } \\
\text { calcite }\end{array}$ & $\begin{array}{c}\text { Mole\%Mg } \\
\text { dolomite }\end{array}$ & $\begin{array}{l}\text { Mole\%Mg } \\
\text { calcite }\end{array}$ & $\underset{\substack{\mathrm{Sr} / \mathrm{Ca} \\
(\mathrm{mol} / \mathrm{mol})}}{ }$ & $\begin{array}{l}\text { Mole\% Fe } \\
\text { calcite }\end{array}$ & $\begin{array}{l}\text { Mole\% Fe } \\
\text { dolomite }\end{array}$ & $\begin{array}{l}\text { Mole\%oMn } \\
\text { calcite }\end{array}$ & $\begin{array}{c}\text { Mole\%Mn } \\
\text { dolomite }\end{array}$ & $\begin{array}{c}\mathrm{Zn} / \mathrm{Ca} \\
(\mathrm{mol} / \mathrm{mol})\end{array}$ & $\mathrm{X}$-ray \\
\hline $2 \mathrm{H}-3,2-3$ & Silty mud/muddy silt & 139301 & 12.32 & -11.36 & -7.79 & 0.705331 & 63.63 & & 32.43 & & $1.54 \mathrm{E}-03$ & & 2.90 & & 1.03 & $3.94 \mathrm{E}-04$ & minor $\mathrm{D}$ \\
\hline $2 \mathrm{H}-3,5-6$ & Silty mud/muddy silt & 139302 & 12.35 & -11.72 & -8.19 & 0.705035 & 71.23 & & 25.20 & & $5.21 \mathrm{E}-04$ & & 2.29 & & 1.28 & $1.07 \mathrm{E}-04$ & $D>L$ \\
\hline $2 \mathrm{H}-3,68$ & Small nodules & 139254 & 12.31 & -10.95 & -7.93 & 0.704994 & 63.97 & & 32.10 & & $6.41 \mathrm{IE}-04$ & & 2.67 & & 1.26 & $9.73 \mathrm{E}-05$ & $D>L$ \\
\hline 2H-3, 15-18 & Poorly indur. mud/ large nodule & 139255 & 12.35 & -10.7 & -7.42 & 0.705352 & & & & & & & & & & & D \\
\hline $\begin{array}{l}2 \mathrm{H}-3.18-19 \\
2 \mathrm{H}-3.21-22\end{array}$ & $\begin{array}{l}\text { Silty mud/muddy sitt } \\
\text { Silty mud/muddy sitt }\end{array}$ & 139303 & 12.48 & -11.57 & -6.4 & 0.705179 & 54.81 & & $\begin{array}{l}40.21 \\
3961\end{array}$ & & $6.70 \mathrm{E}-04$ & & 3.48 & & 1.50 & $1.02 \mathrm{E}-04$ & $\mathrm{D}$ \\
\hline $\begin{array}{l}2 \mathrm{H}-3,21-22 \\
2 \mathrm{H}-3,24-25\end{array}$ & $\begin{array}{l}\text { Silty mud/muddy silt } \\
\text { Silty mud/muddy sitt }\end{array}$ & $\begin{array}{l}139304 \\
139305\end{array}$ & $\begin{array}{l}12.51 \\
12.54\end{array}$ & $\begin{array}{l}-10.62 \\
-10.47\end{array}$ & $\begin{array}{l}-7.03 \\
-6.76\end{array}$ & $\begin{array}{l}0.705073 \\
0.705026\end{array}$ & $\begin{array}{l}55.72 \\
58.76\end{array}$ & & $\begin{array}{l}39.61 \\
37.48\end{array}$ & & $\begin{array}{l}7.87 \mathrm{E}-04 \\
1.33 \mathrm{E}-03\end{array}$ & & $\begin{array}{l}3.38 \\
2.56\end{array}$ & & $\begin{array}{l}1.29 \\
1.20\end{array}$ & $\begin{array}{l}1.19 \mathrm{E}-04 \\
2.97 \mathrm{E}-04\end{array}$ & $\begin{array}{c}\mathrm{D} \\
\mathrm{D}\end{array}$ \\
\hline $2 \mathrm{H}-3,29-30$ & Silty mud/muddy silt & 139306 & $\begin{array}{l}12.54 \\
12.59\end{array}$ & $\begin{array}{l}-10.47 \\
-17.5\end{array}$ & $\begin{array}{l}-6.16 \\
-3.24\end{array}$ & $0 . / 05026$ & $\begin{array}{l}38.46 \\
61.49\end{array}$ & & $\begin{array}{l}37.48 \\
35.05\end{array}$ & & $\begin{array}{l}1.3 \mathrm{SE}-03 \\
2.45 \mathrm{E}-03\end{array}$ & & 2.46 & & $\begin{array}{l}1.20 \\
1.00\end{array}$ & $4.01 \mathrm{E}-04$ & $\operatorname{minor} D$ \\
\hline $2 \mathrm{H}-3,119-120$ & Silty mud/muddy silt & 139307 & 13.49 & -11.79 & -7.7 & 0.704816 & 69.45 & & 27.62 & & $9.90 \mathrm{E}-04$ & & 2.19 & & 0.73 & $1.81 \mathrm{IE}-04$ & D $>H$ \\
\hline $2 \mathrm{H}-3,124-125$ & Silty mud/muddy silt & 139308 & 13.54 & -12.55 & -9.41 & & 65.13 & & 31.67 & & $1.14 \mathrm{E}-03$ & & 2.43 & & 0.77 & $2.55 \mathrm{E}-04$ & $D>L$ \\
\hline $\begin{array}{l}2 \mathrm{H}-3,129-130 \\
2 \mathrm{H}-4,40-42\end{array}$ & $\begin{array}{l}\text { Silty mud/muddy silt } \\
\text { Small }\end{array}$ & 139309 & 13.59 & $\begin{array}{r}-12.19 \\
-1697\end{array}$ & -9.3 & 0.705159 & 66.21 & & 30.57 & & $8.84 \mathrm{E}-04$ & & 2.40 & & 0.81 & $1.62 \mathrm{E}-04$ & $D>L$ \\
\hline $\begin{array}{l}2 \mathrm{H}-4,40-42 \\
2 \mathrm{H}-4,50-52\end{array}$ & $\begin{array}{l}\text { Small poorly indur. nodules/clumped silt } \\
\text { Silt/mud }\end{array}$ & $\begin{array}{l}139259 \\
139260\end{array}$ & $\begin{array}{l}14.2 \\
14.3\end{array}$ & $\begin{array}{l}-16.97 \\
-1675\end{array}$ & $\begin{array}{l}-17.26 \\
-1704\end{array}$ & 0.704485 & & 95.75 & & $\begin{array}{l}2.88 \\
2.64\end{array}$ & $\begin{array}{l}2.65 \mathrm{E}-04 \\
2.62 \mathrm{E}-04\end{array}$ & 0.40 & & $\begin{array}{l}0.97 \\
1.04\end{array}$ & & $5.73 \mathrm{E}-05$ & L \\
\hline $2 \mathrm{H}-4,55-56$ & $\begin{array}{l}\text { Nodule } \\
\text { Noll }\end{array}$ & 139261 & 14.35 & -16.84 & $\begin{array}{l}-17.04 \\
-17.18\end{array}$ & & & 96.69 & & $\begin{array}{l}2.04 \\
1.99\end{array}$ & $\begin{array}{l}2.020-04 \\
1.40 \mathrm{E}-04\end{array}$ & 0.18 & & 1.14 & & $\begin{array}{l}-174 \mathrm{E}-03 \\
2.17 \mathrm{E}-05\end{array}$ & $\mathrm{~L}$ \\
\hline $2 \mathrm{H}-4,94-97$ & Mud/silt & 139263 & 14.7 & -16.62 & -16 & & & 95.90 & & 2.80 & $2.37 \mathrm{E}-04$ & 0.23 & & 1.07 & & $5.55 \mathrm{E}-05$ & L \\
\hline $2 \mathrm{H}-5,78-80$ & Clumped silt/mud & 139266 & 16.08 & -19.07 & -18.76 & & & 95.48 & & 3.11 & $1.58 \mathrm{E}-04$ & 0.11 & & 1.31 & & $3.67 \mathrm{E}-05$ & $\mathrm{~L}$ \\
\hline $\begin{array}{l}2 \mathrm{H}-5,82-83 \\
4 \mathrm{H}-2,18-20\end{array}$ & $\begin{array}{l}\text { Silt/mud } \\
\text { Soduren }\end{array}$ & 139267 & 16.12 & -19.2 & -19.1 & 0706080 & & 95.77 & & 2.77 & $1.64 \mathrm{E}-04$ & 0.13 & & 1.33 & & $5.02 E-05$ & $\mathrm{~L}$ \\
\hline $\begin{array}{l}4 \mathrm{H}-2,18-20 \\
4 \mathrm{H}-2,48-50\end{array}$ & $\begin{array}{l}\text { Nodule (burrow fill) } \\
\text { Nodule }\end{array}$ & $\begin{array}{l}139271 \\
139272\end{array}$ & 20.5 & $\begin{array}{l}-17.58 \\
-27.77\end{array}$ & $\begin{array}{r}-12.54 \\
1.88\end{array}$ & 0.706089 & & $\begin{array}{l}91.26 \\
92.66\end{array}$ & & $\begin{array}{l}5.56 \\
6.41\end{array}$ & $\begin{array}{l}\begin{array}{l}2.35 \mathrm{E}-04 \\
3.89 \mathrm{E}-04\end{array} \\
-\end{array}$ & 2.20 & & 0.89 & & $\begin{array}{l}2.4 \mathrm{E}-03 \\
1.99 \mathrm{E}-05\end{array}$ & $\underset{\mathrm{H}}{\mathrm{H}}$ \\
\hline $4 \mathrm{H}-2,52-54$ & Nodule & 139274 & 20.54 & -28.02 & 2.55 & & & & & & & & & & & & \\
\hline $4 \mathrm{H}-2,58-60$ & Nodule & 139277 & 20.62 & -18.51 & -7.19 & & & 90.31 & & 5.09 & $2.41 \mathrm{E}-04$ & 3.25 & & 1.35 & & 2.15E-05 & $\mathrm{H}$ \\
\hline $4 \mathrm{H}-2,83-84$ & $\begin{array}{l}\text { Nodule } \\
\text { Sogle }\end{array}$ & 139278 & 20.87 & -18.4 & -7.31 & & & & & & & & & & & $2.49 \mathrm{E}-05$ & $\mathrm{H}$ \\
\hline 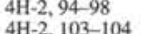 & $\begin{array}{l}\text { Nodule } \\
\text { Small pule (burrow fill }\end{array}$ & $\begin{array}{l}139279 \\
139280\end{array}$ & $\begin{array}{l}20.98 \\
2107\end{array}$ & -33.96 & $\begin{array}{r}2.69 \\
-1094\end{array}$ & & & $\begin{array}{l}88.01 \\
89.37 \\
89\end{array}-10$ & & $\begin{array}{l}11.92 \\
702\end{array}$ & $5.40 \mathrm{E}-04$ & 0.02 & & 0.04 & & $1.95 \mathrm{E}-05$ & $\mathrm{H}_{\mathrm{H}}$ \\
\hline $\begin{array}{l}4 \mathrm{H}-2,103-104 \\
4 \mathrm{H}-2,109-110\end{array}$ & $\begin{array}{l}\text { Small nodule (burrow fill) } \\
\text { Small nodule (burrow fill) }\end{array}$ & $\begin{array}{l}\begin{array}{l}139280 \\
139281\end{array} \\
\end{array}$ & $\begin{array}{l}21.07 \\
21.12\end{array}$ & $\begin{array}{l}-17.72 \\
-19.46\end{array}$ & $\begin{array}{l}-10.94 \\
-17.58\end{array}$ & & & $\begin{array}{l}89.37 \\
94.77\end{array}$ & & $\begin{array}{l}7.02 \\
3.96\end{array}$ & $\begin{array}{l}3.11 \mathrm{IE}-04 \\
2.05 \mathrm{E}-04\end{array}$ & $\begin{array}{l}2.41 \\
0.06\end{array}$ & & $\begin{array}{l}1.20 \\
1.21\end{array}$ & & $\begin{array}{l}3.12 \mathrm{E}-05 \\
1.37 \mathrm{E}-04\end{array}$ & $\underset{L}{\mathrm{H}}$ \\
\hline $4 \mathrm{H}-2,126-127$ & Small nodule (burrow fill) & 139283 & 21.3 & -18.7 & -7.59 & & & 89.50 & & 5.82 & $2.64 \mathrm{E}-04$ & 3.49 & & 1.19 & & 3.63E-05 & $\mathrm{H}$ \\
\hline $4 \mathrm{H}-2,148-150$ & Nodulc & 139285 & 21.52 & -33.89 & 2.39 & & & 92.54 & & 7.35 & 3.45E-04 & 0.02 & & 0.09 & & $2.13 \mathrm{E}-05$ & $\mathrm{H}$ \\
\hline $4 \mathrm{H}-3,15-17$ & Nodule & 139286 & 21 & -19.51 & -18.78 & 070503 & & 95.90 & & 2.85 & $1.40 \mathrm{E}-04$ & 0.09 & & 1.17 & & $3.08 \mathrm{E}-05$ & $\mathrm{~L}$ \\
\hline $\begin{array}{l}4 \mathrm{H}-3,0-2 \\
4 \mathrm{H}, 0-150\end{array}$ & $\begin{array}{l}\text { Nodule } \\
\text { Small nodule (burrow fill) }\end{array}$ & $\begin{array}{l}139991 \\
139292\end{array}$ & ${ }_{23.04}^{23}$ & $\begin{array}{l}-29.47 \\
-34.22\end{array}$ & $\begin{array}{l}2.46 \\
2.66\end{array}$ & 0.70593 & & $\begin{array}{l}93.05 \\
92.76\end{array}$ & & $\begin{array}{l}6.42 \\
7.12\end{array}$ & $\begin{array}{l}3.800-04 \\
3.54 \mathrm{E}-04\end{array}$ & $\begin{array}{l}0.02 \\
0.03\end{array}-1$ & & $\begin{array}{l}0.50 \\
0.09\end{array}$ & & $\begin{array}{l}1.68 \mathrm{E}-05 \\
1.87 \mathrm{E}-05\end{array}$ & ${ }_{\mathrm{H}}^{\mathrm{H}}$ \\
\hline
\end{tabular}

Note: All concentrations are reported in units of mole\% of the carbonate fraction or as mol/mol ratios (in the case of strontium and zinc). The last column contains the results of qualitative $\mathrm{X}$-ray diffraction of the carbonate phases. $\mathrm{H}=$ high-magnesium calcite; $\mathrm{L}=\mathrm{low}$-magnesium calcite; $\mathrm{D}=$ dolomite. An asterisk identifies sub-samples that have been combined into one sample for the purposes of $\mathrm{X}$-ray diffraction. 


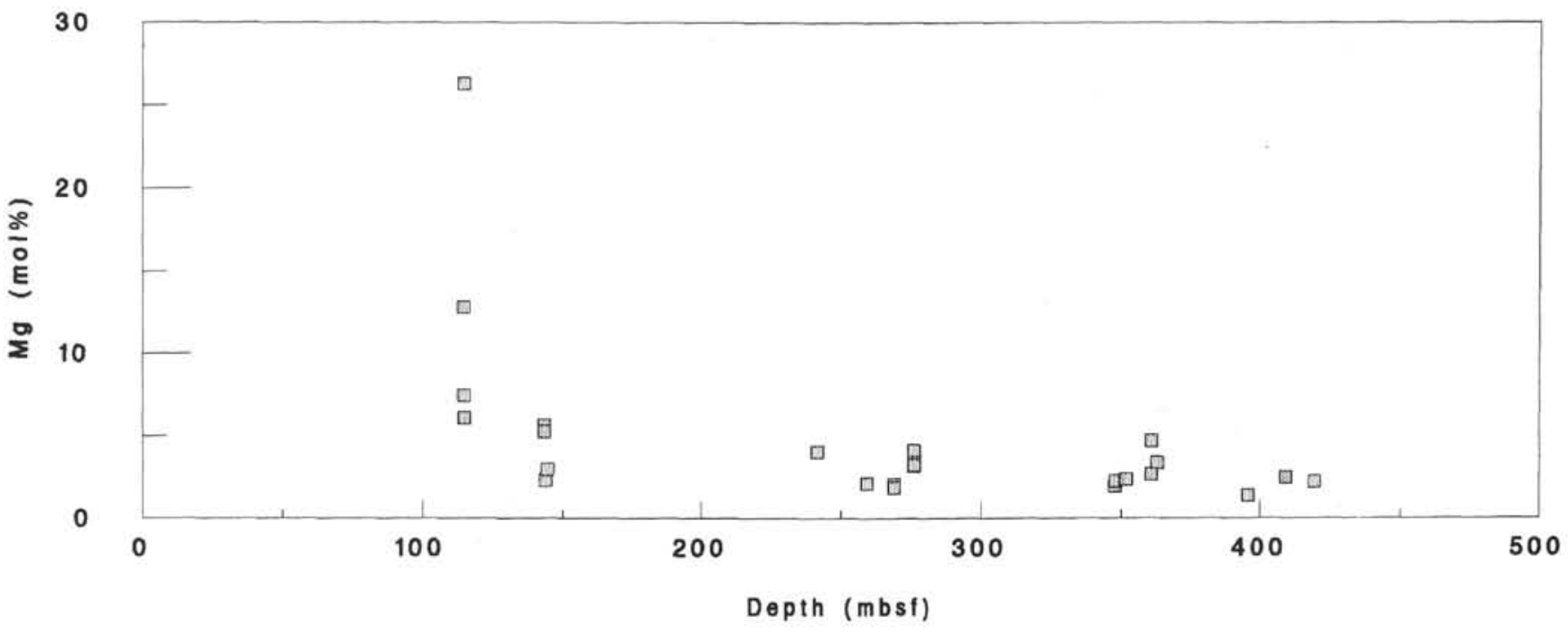

Figure 3. Magnesium contents of calcites vs. depth for Hole 857C.

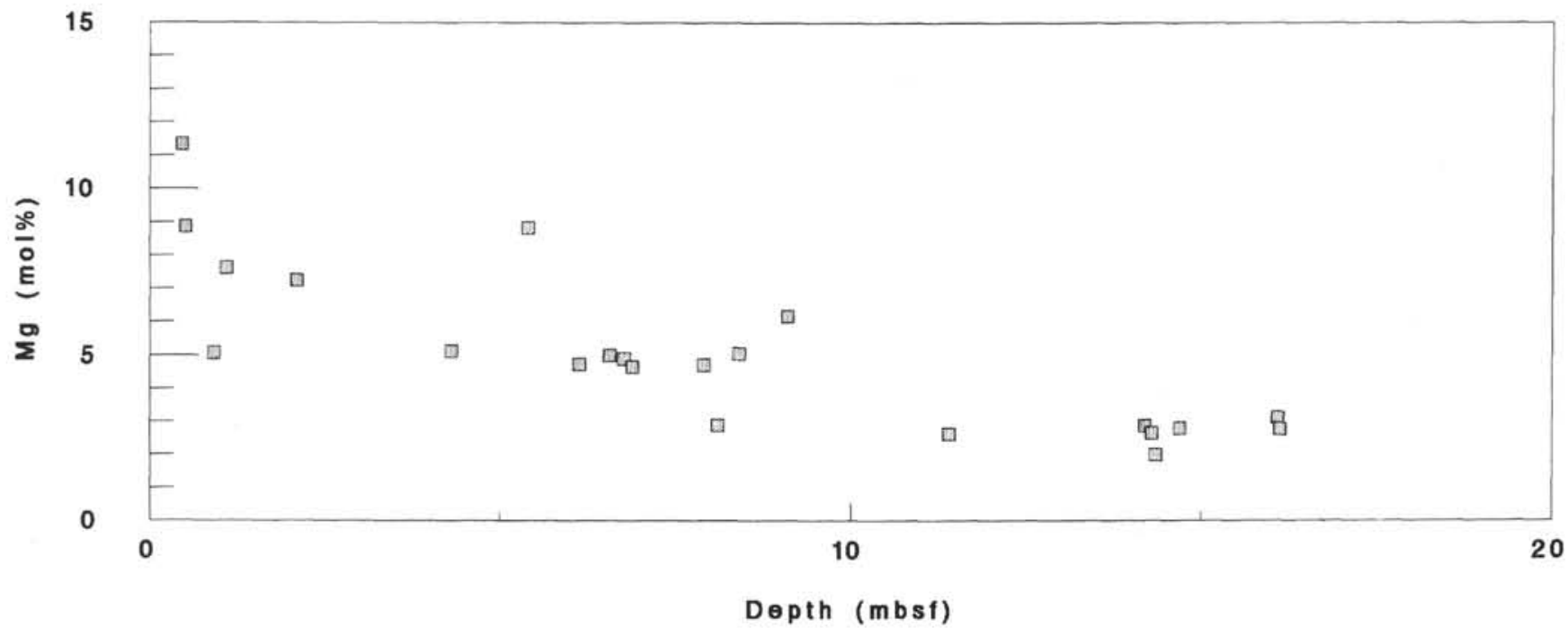

Figure 4. Magnesium contents of calcites vs. depth for Cores $858 \mathrm{D}-1 \mathrm{H}$ and $-2 \mathrm{H}$.

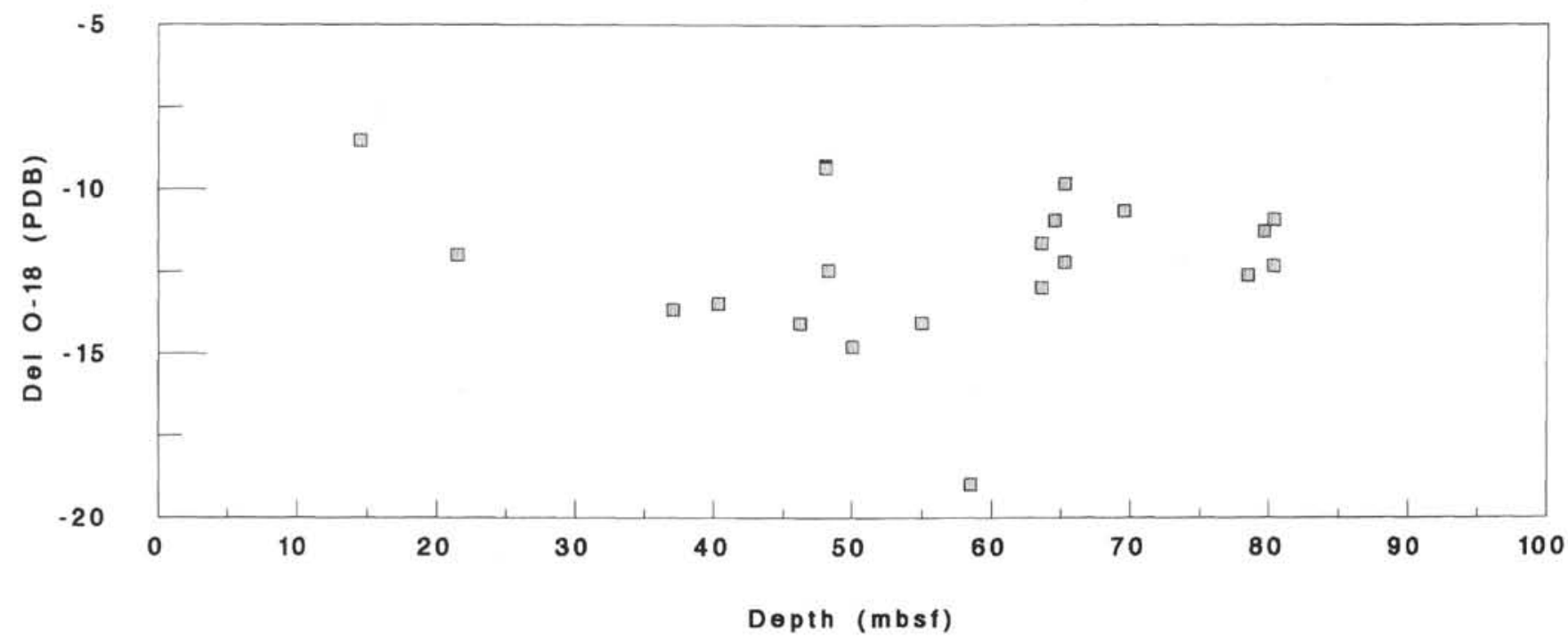

Figure 5. Oxygen isotopic composition of calcites vs. depth for Hole 856A. Reported as \%o relative to the PDB standard. 
P.A. BAKER, S.L. CROSS, S.J. BURNS

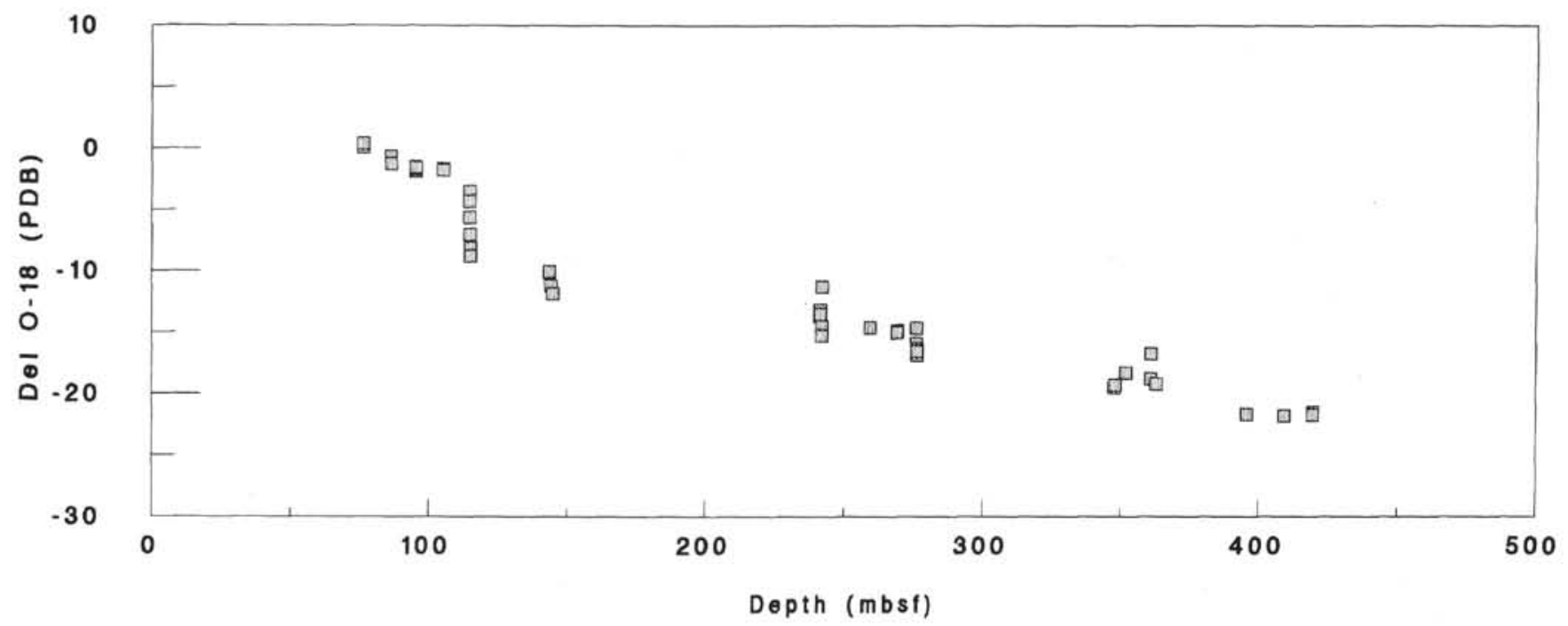

Figure 6. Oxygen isotopic composition of dolomites and calcites vs. depth for Hole 857C.

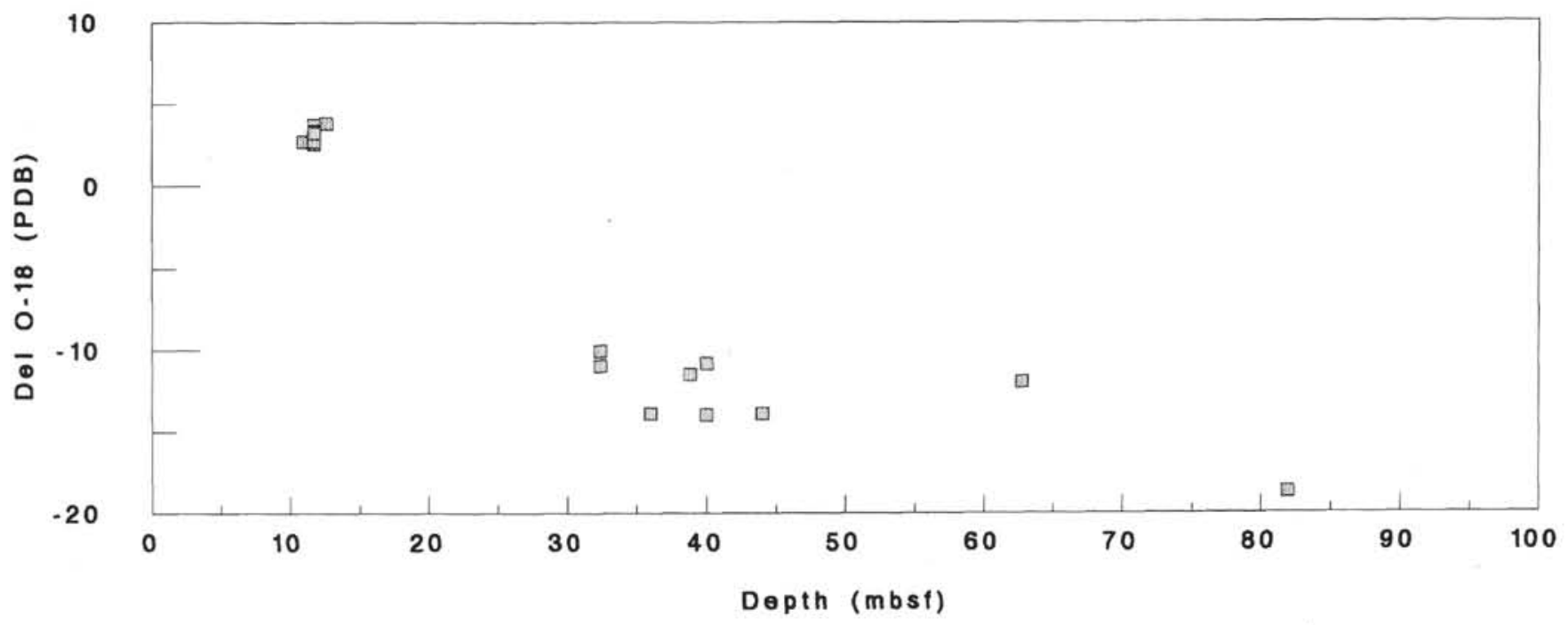

Figure 7. Oxygen isotopic composition of calcites vs. depth for Hole 858A.

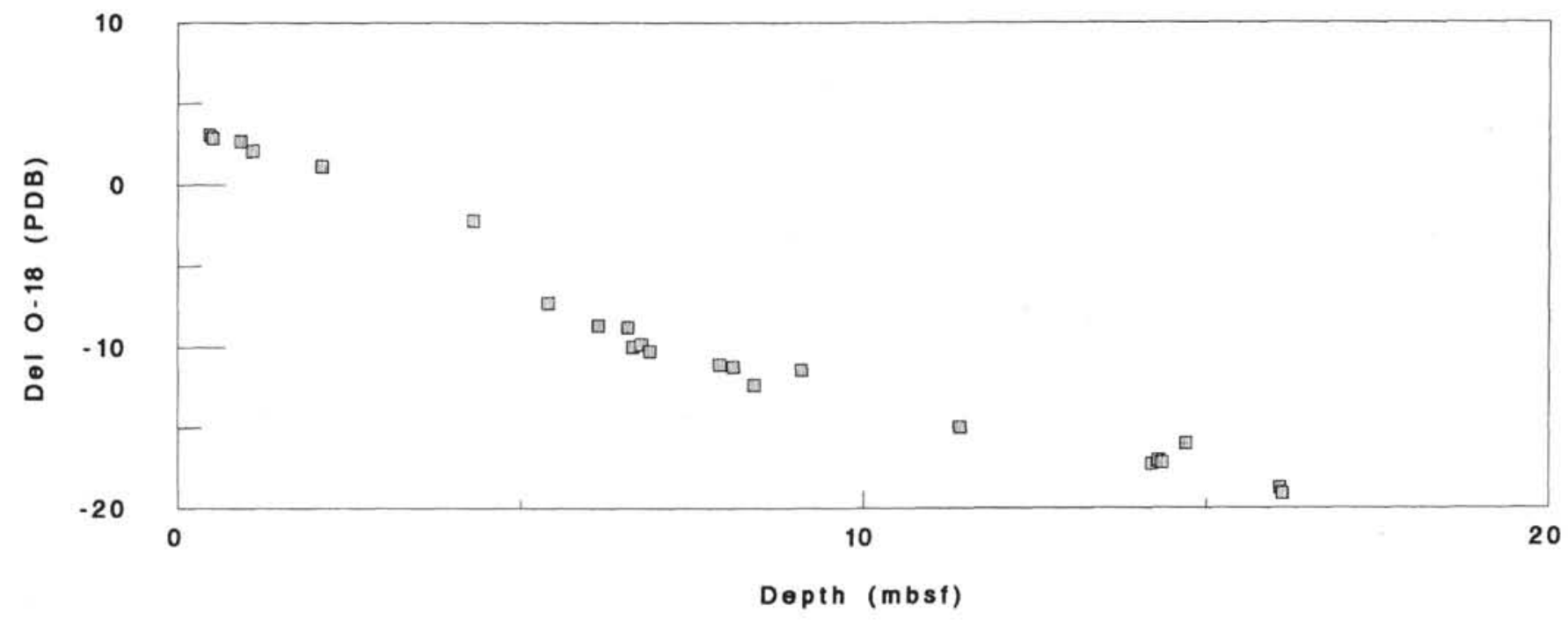

Figure 8. Oxygen isotopic composition of calcites vs. depth for Cores $858 \mathrm{D}-1 \mathrm{H}$ and $-2 \mathrm{H}$. 


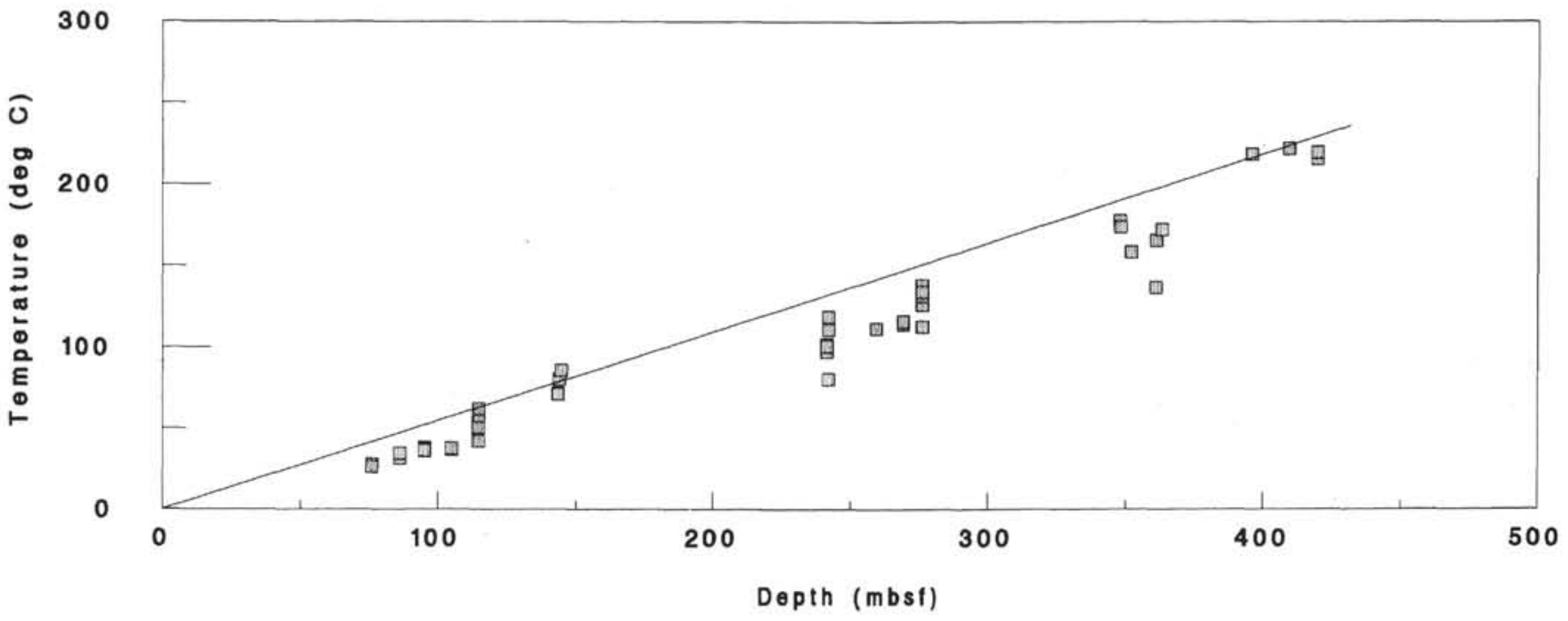

Figure 9. Calculated temperatures vs. depth for Hole 857C. The line intercepts bottom-water temperature at the sediment-water interface and passes through the hottest values at any given depth. Its slope is $0.53^{\circ} \mathrm{C} / \mathrm{m}$.

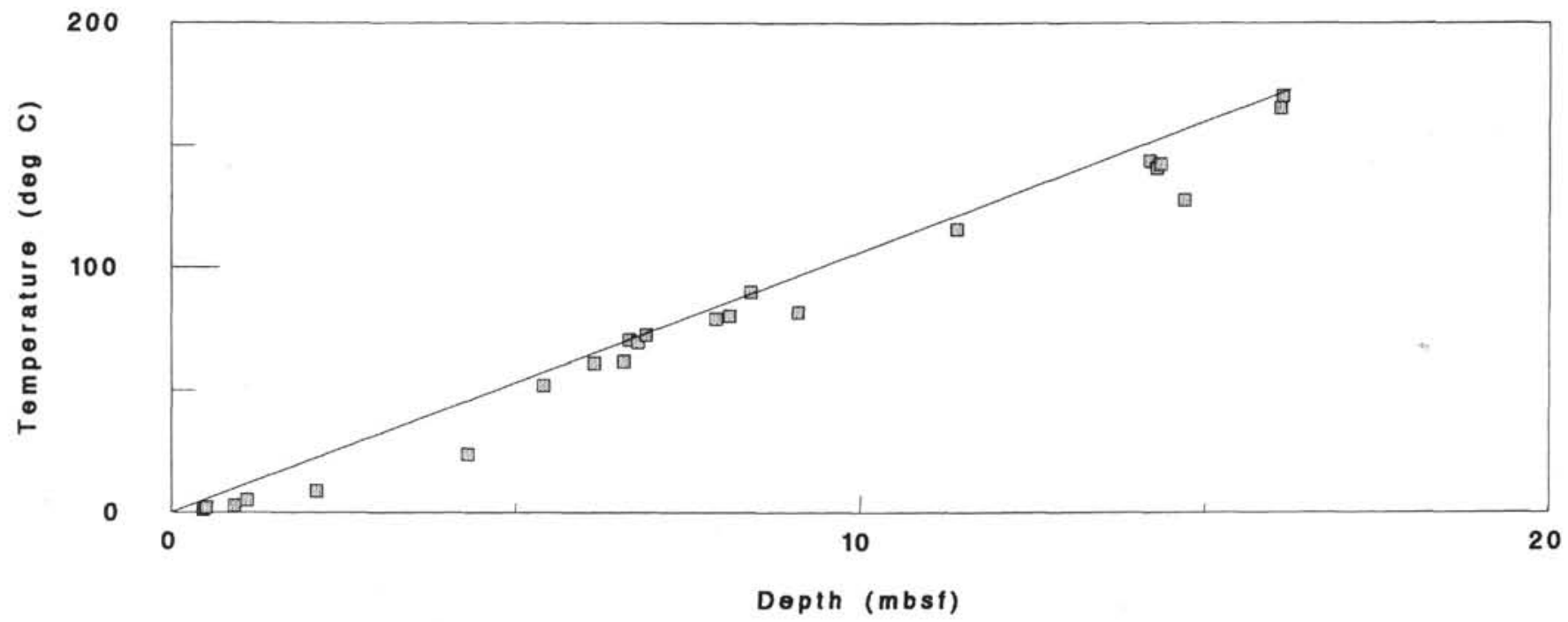

Figure 10. Calculated temperatures vs. depth for Cores $858 \mathrm{D}-1 \mathrm{H}$ and $-2 \mathrm{H}$. The line intercepts bottom-water temperature at the sediment-water interface and passes through the hottest values at any given depth. Its slope is $10.5^{\circ} \mathrm{C} / \mathrm{m}$.

$858 \mathrm{D}$ and about $0.53^{\circ} \mathrm{C} / \mathrm{m}$ at Hole $857 \mathrm{C}$. These data are consistent with an interpretation of conductive heat flow within the sediment column in both holes indicating that the carbonates apparently remain in or near isotopic equilibrium with the pore fluids at the present-day burial depths. Although there is less adequate data coverage, a significant number of samples from Hole 858A indicate temperatures hotter than predicted by the present-day geotherm.

Hole $856 \mathrm{~A}$ has had a more complicated thermal history than the other analyzed sections. The shallowest carbonate sample analyzed, a small nodule from $14.5 \mathrm{mbsf}$, records a temperature of $60^{\circ} \mathrm{C}$, the lowest temperature calculated for any of the samples from this hole. The present-day temperature at this burial depth is estimated to be about $10^{\circ} \mathrm{C}$ (Davis, Mottl, Fisher, et al., 1992). Although the age of the sediment at 14.5 mbsf is not known, we conclude that hydrothermal activity associated with emplacement of mafic sills and, possibly, with formation of the nearby massive sulfide ore body (Davis, Mottl, Fisher, et al, 1992), affected nearly the entire stratigraphic column; therefore alteration was very recent. The lack of a significant downhole temperature gradient is probably best explained by carbonate

Table 2. Comparison of calculated and measured downhole temperatures.

\begin{tabular}{lrrrr}
\hline Hole & $\begin{array}{c}\delta^{18} \mathrm{O} \\
(\text { SMOW })\end{array}$ & $\begin{array}{c}\text { Depth } \\
(\mathrm{m})\end{array}$ & $\begin{array}{c}\mathrm{T}_{\mathrm{c}} \\
\left({ }^{\circ} \mathrm{C}\right)\end{array}$ & $\begin{array}{c}\mathrm{T}_{\mathrm{m}} \\
\left({ }^{\circ} \mathrm{C}\right)\end{array}$ \\
\hline $856 \mathrm{~A}$ & 11.34 & 58 & 169 & 31 \\
$857 \mathrm{C}$ & 8.45 & 409 & 222 & 224 \\
$858 \mathrm{~A}$ & 11.60 & 82 & 165 & 155 \\
$858 \mathrm{D}$ & 11.22 & 16 & 171 & 160
\end{tabular}

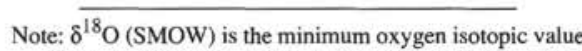
for a carbonate sample at each hole. The sub-bottom depth of that sample is given in the third column. $T_{c}=$ temperature calculated from the isotopic composition of the sample. $\mathrm{T}_{\mathrm{m}}=$ temperature calculated at that sample depth from downhole measurements. 


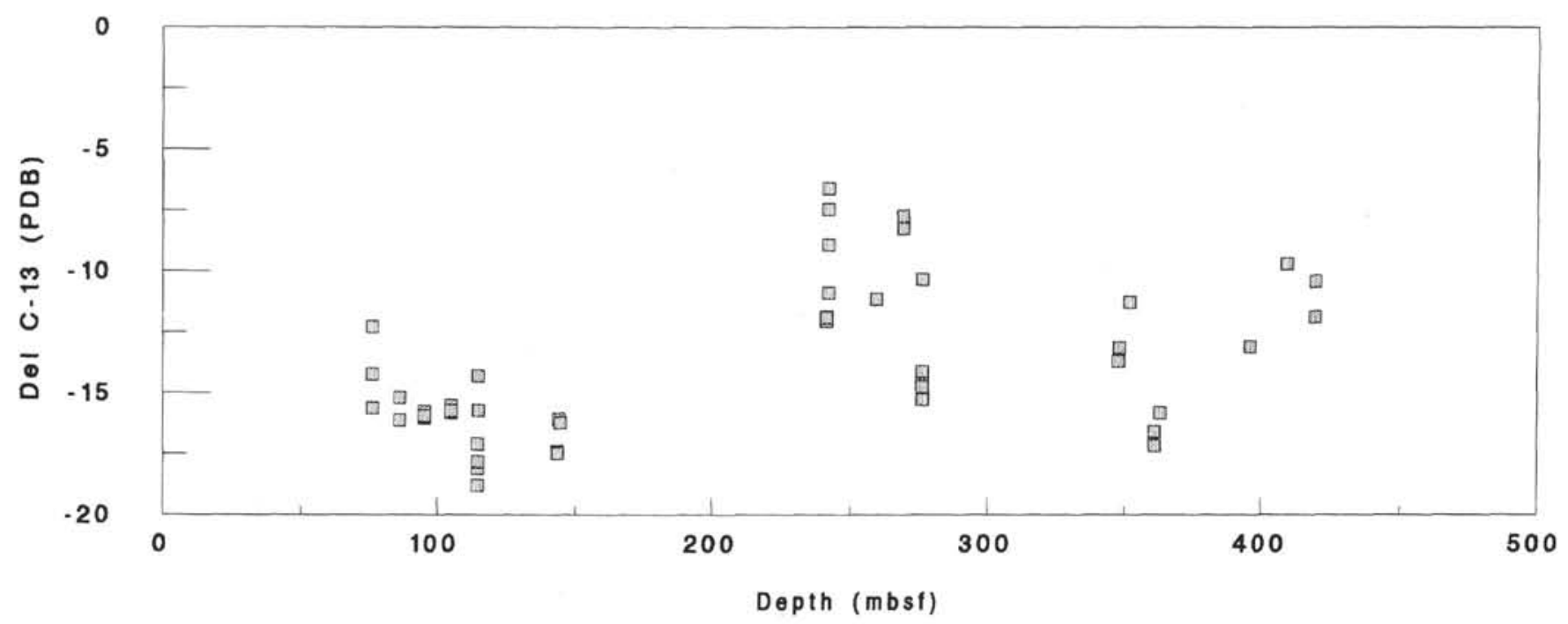

Figure 11. Carbon isotopic composition of dolomites and calcites vs. depth for Hole 857C.

recrystallization. We hypothesize that original oxygen isotopic compositions of carbonates precipitated prior to hydrothermal activity were reset to varying degrees during this recent event.

\section{CARBON ISOTOPES AND CARBON SOURCES}

One of the important results of this study is identification of the various carbon sources to the authigenic carbonates. To the extent that these carbonates are a product of hydrothermal activity, the carbon sources of the nodules reflect the carbon sources of the hydrothermal fluid. Because carbon is one of the most abundant redox-sensitive or redox-controlling elements in the hydrothermal fluids of Middle Valley, its chemistry is of great interest. Discussion will focus on the two holes with the most complete data sets.

Hole $857 \mathrm{C}$ (Fig. 11) displays features typical of authigenic carbonates from many organic-rich sedimentary profiles previously analyzed (e.g., Claypool and Kaplan, 1974; Kelts and McKenzie, 1982; Baker and Burns, 1985). Carbon is buried in the form of biogenic calcite $\left(\delta^{13} \mathrm{C} \approx 0 \%\right.$ PDB ) and organic carbon $\delta^{13} \mathrm{C} \approx-20 \%$ PDB $)$. Throughout the upper 50 to $100 \mathrm{mbsf}$ of the hole, microbial sulfate reduction results in an input to the pore waters of oxidized organic carbon, mostly in the form of $\mathrm{HCO}_{3}^{-}\left(\delta^{13} \mathrm{C} \approx-20 \%\right.$ PDB $)$. Because of the steep thermal gradients at this site, microbial sulfate reduction is probably succeeded by thermocatalytic sulfate reduction over the depth range of 100 to 200 mbsf. Over the same depth range, it is likely that pore water remains near equilibrium with respect to anhydrite (Davis, Mottl, Fisher, et al., 1992). Sulfate reduction is complete (below approximately $200 \mathrm{mbsf}$ ) when dissolved sulfate is almost totally depleted in pore fluids and when anhydrite is totally dissolved from the sediments. Again, because of the relatively high thermal gradient, thermal maturation of sedimentary organic carbon becomes important at these relatively shallow burial depths and thermocatalytic decarboxylation results in an input of isotopically depleted $\left(\delta^{13} \mathrm{C} \approx-20 \%\right.$ dissolved $\mathrm{CO}_{2}$ to pore fluids.

One unusual aspect of this pattern of organic diagenesis is the absence of a zone of abundant biogenic methane. In previously analyzed continental-margin sedimentary successions, methanogenesis often imparts a positive carbon isotopic signature to authigenic carbonates. In the present sediments, most of the methane in the pore fluids appears to be derived from thermal cracking.

When the temperature-dependent fractionation factors for carbon isotopes between dolomite and calcite (Sheppard and Schwarcz, 1970) and between calcite and dissolved bicarbonate (Mook et al., 1974 and Malinin et al. reported by Friedman and O'Neil, 1977) are applied, we calculate that the carbon isotopic composition of dissolved bicarbonate at Hole $857 \mathrm{C}$ is surprisingly uniform throughout the entire hole, ranging from about $-3 \%$ to $-13 \%$, but overwhelmingly $-10 \pm 2 \%$. This suggests a roughly equal contribution of carbon to the pore fluids from the oxidation of organic carbon and from the recrystallization of biogenic calcite.

The depth variation of $\delta^{13} \mathrm{C}$ in authigenic carbonates in Hole 858D (Cores $1 \mathrm{H}$ and $2 \mathrm{H}$ ) is shown in Figure 12. Applying the appropriate equilibrium fractionation factors, we conclude that pore water bicarbonate in equilibrium with the authigenic carbonates has $\delta^{13} \mathrm{C} \approx-20 \%$ PDB between 5 and 20 mbsf and decreases to values of about $-38 \%$ PDB just below the sediment-water interface. Thus, the deeper pore waters in this hole, which resemble modern vent fluids in many of their chemical characteristics (Butterfield et al., this volume), contain carbon that is almost completely derived from the oxidation of sedimentary organic carbon. At depths less than $5 \mathrm{mbsf}$, methane oxidation during microbial sulfate reduction results in a more negative carbon isotopic composition of dissolved bicarbonate. As noted above, methane oxidation maintains the high degree of carbonate supersaturation in the pore waters necessary to allow the formation of HMC. Very negative carbon isotopic signals were also observed in carbonates from the upper 13 mbsf of Hole $858 \mathrm{~A}$, at $12.8 \mathrm{mbsf}$ in Hole $858 \mathrm{C}$, and in recrystallized LMC samples at $48 \mathrm{mbsf}$ in Hole $856 \mathrm{~A}$. Goodfellow and Blaise (1988) and Al-Aasm and Blaise (1991) have previously reported similar isotopic compositions of carbonates recovered by traditional piston coring within the bounds of the active vent field.

\section{STRONTIUM AND STRONTIUM ISOTOPES}

Strontium in carbonates in the Middle Valley comes from four main sources: seawater $\left({ }^{87} \mathrm{Sr} /{ }^{86} \mathrm{Sr} \sim 0.7091\right)$; biogenic marine carbonate sediments $\left({ }^{87} \mathrm{Sr} /{ }^{86} \mathrm{Sr} \sim 0.709\right.$ ); detrital sediments (strontium isotopic ratios not yet measured); and oceanic basement rocks $\left({ }^{87} \mathrm{Sr} /{ }^{86} \mathrm{Sr} \sim 0.703\right)$.

In Hole $857 \mathrm{C}$ the ${ }^{87} \mathrm{Sr} /{ }^{86} \mathrm{Sr}$ ratios of the carbonates decrease with increasing burial depth (Fig. 13). We observe a better correlation with $\delta^{18} \mathrm{O}$ than with burial depth (this is expected, because most of these samples formed at a somewhat shallower burial depth than the one at which they presently occur). The shallowest carbonates analyzed approach marine values of ${ }^{87} \mathrm{Sr} /{ }^{86} \mathrm{Sr}$, indicating a probable marinecarbonate source of shallow-pore-water strontium. Linear extrapolation of the trend in Figure 13 leads to an isotopic ratio of 0.703 at a depth of about $600 \mathrm{mbsf}$. This is nearly coincident with the depth interval (610 to $615 \mathrm{mbsf}$ ) at which high permeabilities were inferred 


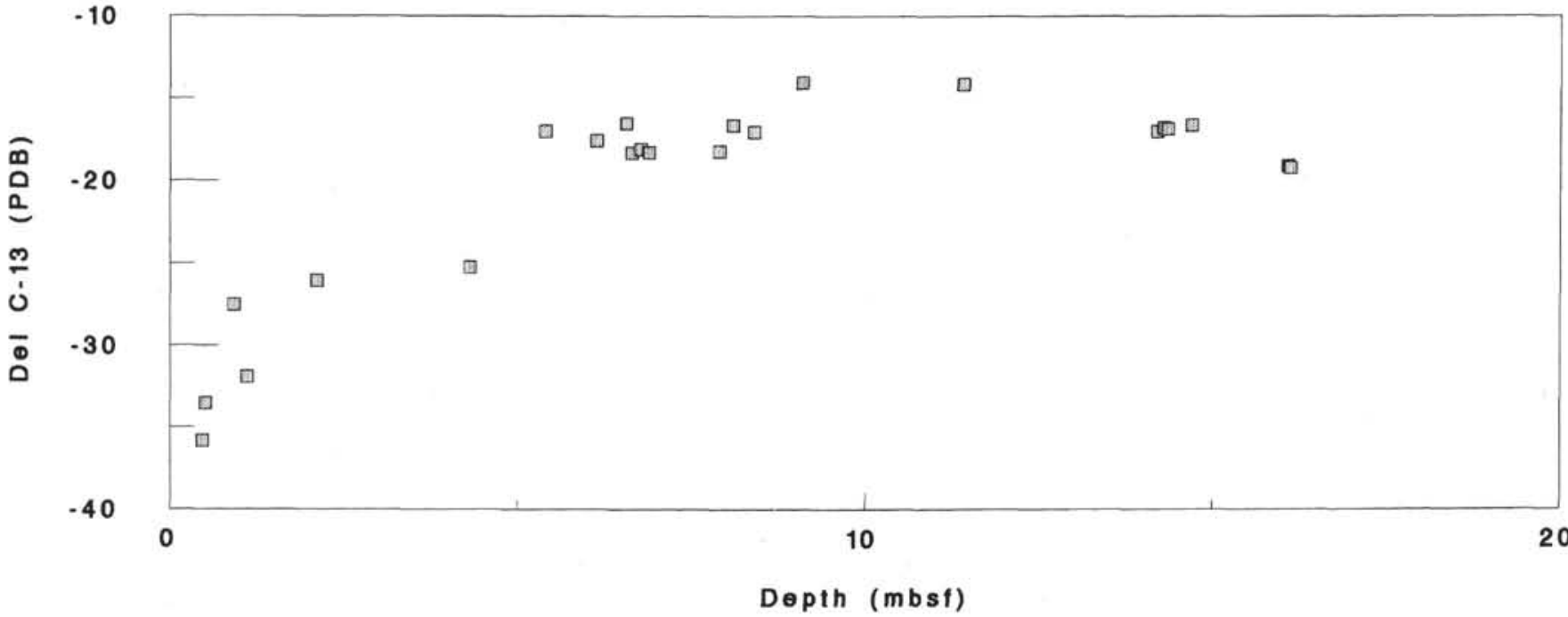

Figure 12. Carbon isotopic composition of dolomites and calcites vs. depth for Cores $858 \mathrm{D}-1 \mathrm{H}$ and $-2 \mathrm{H}$.

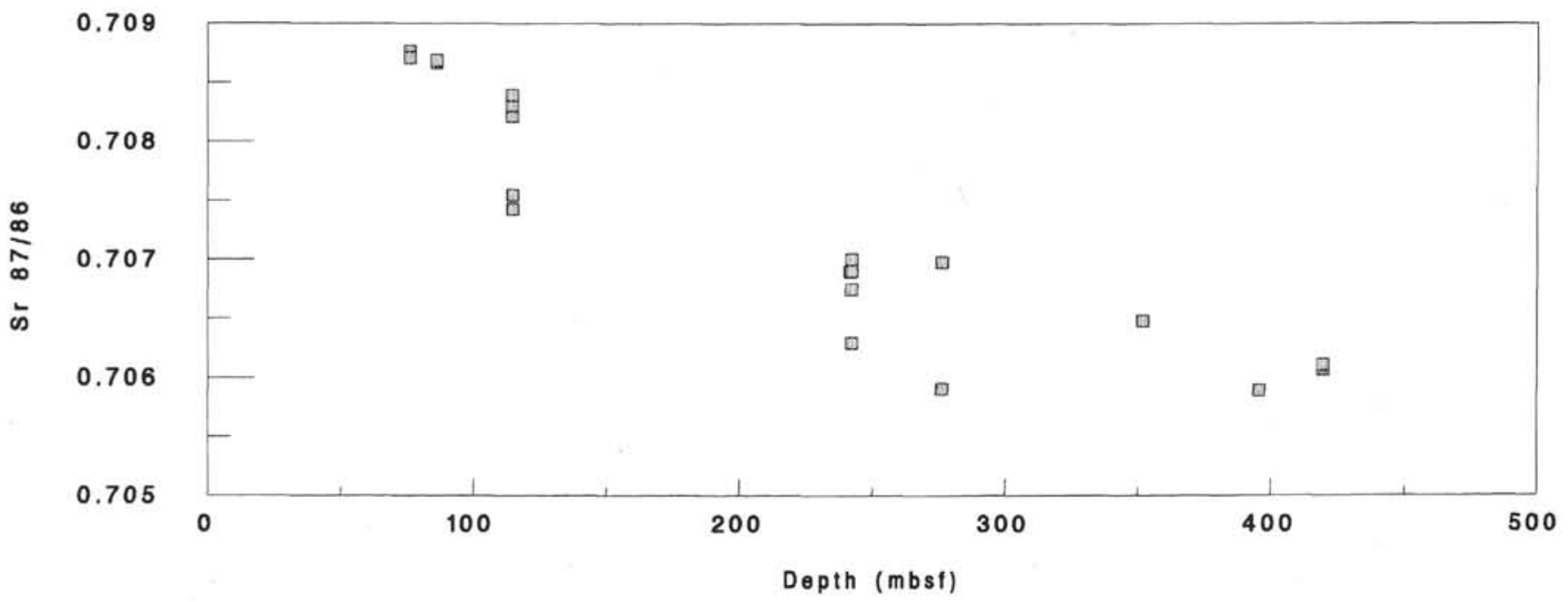

Figure 13. Strontium isotopic composition of calcites and dolomites vs. depth for Hole 857C.

from packer experiments (Davis, Mottl, Fisher, et al., 1992, p. 373). Our interpretation of these data is that pore water at Hole $857 \mathrm{C}$ contains strontium that is a mixture (by the mechanism of diffusion) of seawater strontium and basaltic strontium. Furthermore, the pore fluids within basement in the permeable zone are a pure basaltic endmember with respect to their strontium isotopic composition.

Many of the igneous rocks recovered at Site 857 are heavily altered as is indicated by an increase in wt $\% \mathrm{MgO}$ (see Davis, Mottl, Fisher, et al., 1992, p. 345). In almost all cases, alteration also involves an equivalent loss of calcium and, often, strontium (see Davis, Mottl, Fisher, et al., 1992, table 26, p. 371). This alteration is the likely source of basaltic strontium to basement pore fluids.

In Hole $858 \mathrm{D}$, Cores $1 \mathrm{H}$ and $2 \mathrm{H},{ }^{87} \mathrm{Sr} /{ }^{86} \mathrm{Sr}$ ratios (Fig. 14) vary within the range of 0.7062 in the shallowest sample $(0.5 \mathrm{mbsf})$ to 0.7045 (14.2 mbsf). In the deeper samples, it appears that up to $75 \%$ of the strontium is of basaltic origin and the remainder is of marine origin. Similar values are also found in carbonates from Holes $856 \mathrm{~A}$ and $858 \mathrm{~A}$. In Hole $858 \mathrm{~A}$ the three samples analyzed display increasing strontium isotopic compositions with increasing depth. We tentatively attribute the higher isotopic ratios to the downward or lateral advection of seawater along the flanks of hydrothermal discharge zones and partial entrainment of these fluids as they heat and mix. This hypothesis merits further investigation.

The strontium concentration of diagenetic carbonates is a function of the strontium-to-calcium ratio of pore water solutes and the distribution coefficient $\left(\mathrm{k}_{\mathrm{sr}}\right)$ of strontium in calcite (or dolomite, if appropriate). The distribution coefficients are somewhat temperature-dependent (Baker, unpubl. data), and may also be dependent on rate of precipitation (e.g., Lorens, 1981) and the magnesium content of the calcite (e.g., Mucci and Morse, 1983).

In Hole 857C, the strontium content of calcites increases downcore to a maximum at about $350 \mathrm{mbsf}$ and then decreases in deeper samples (Fig. 15). This peak is similar to the peak in dissolved strontium concentration that spans an interval from about 275 to 300 mbsf (Wheat and Mottl, this volume). The offset between the solid peak and the solute peak may attest to the "memory" of carbonate nodules as they successively accrete carbonate from pore waters of varying composition (assuming a steady-state distribution of dissolved strontium).

In Hole 858D, the strontium contents of calcites (Fig. 16) are not closely related to equilibrium values predicted from pore water chem- 


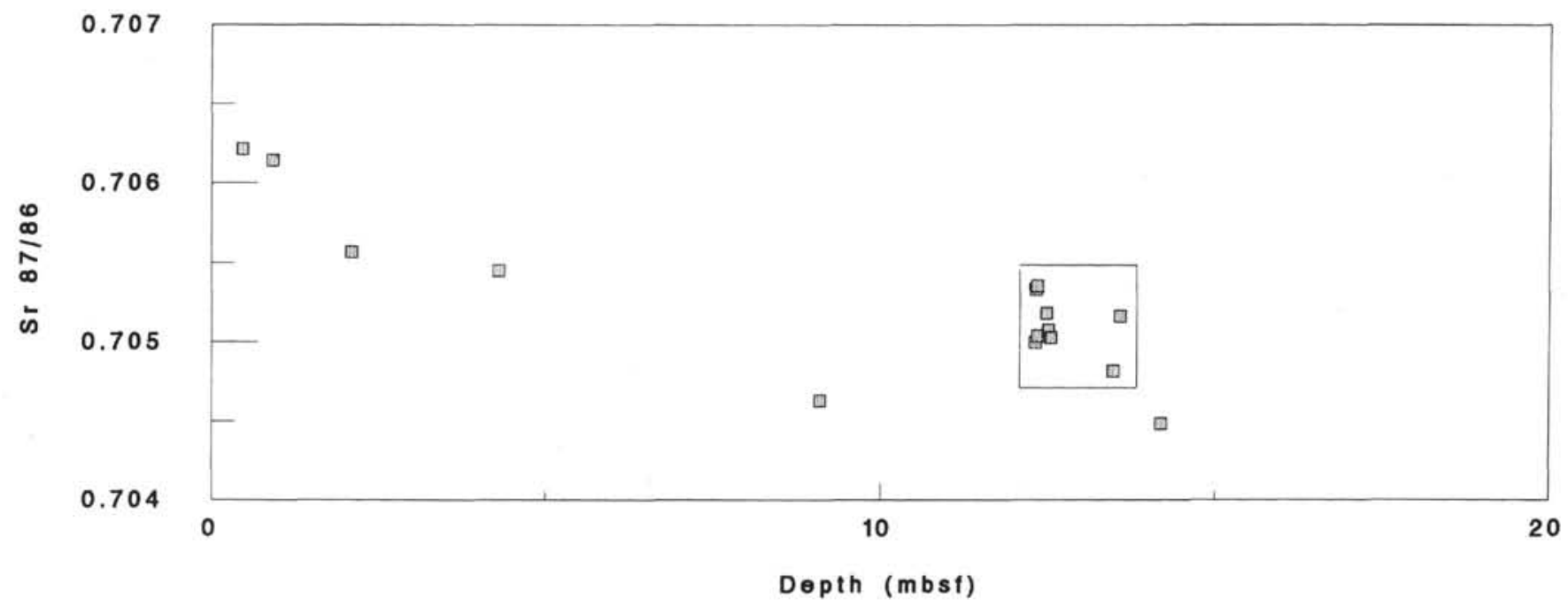

Figure 14. Strontium isotopic composition of calcites and dolomites vs. depth for Hole 858D. Dolomites are outlined by the box. Their elevated values relative to calcites are believed to be due to retention of strontium during dolomitization of pre-existing calcite.

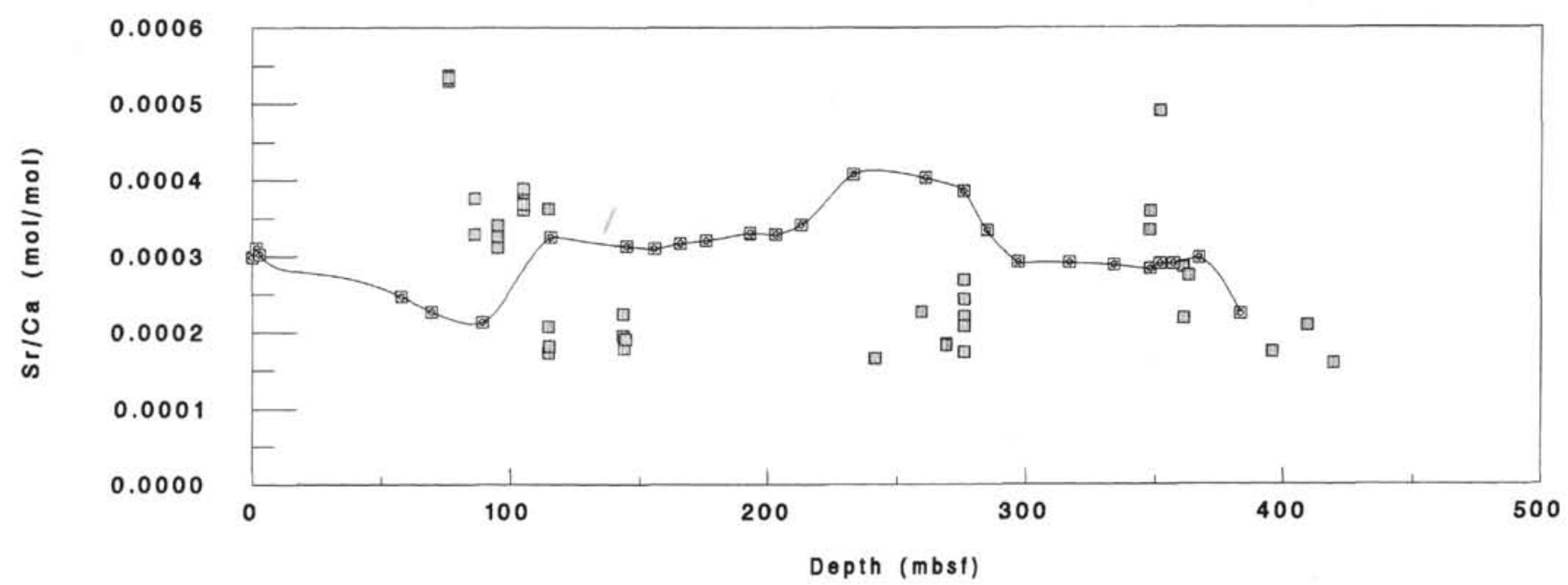

Figure 15. Strontium/calcium ratios of calcites vs. depth for Hole $857 \mathrm{C}$. The squares connected by the line are the calculated equilibrium strontium/calcium ratios using the pore water strontium/calcium ratios from Wheat and Mottl (this volume) and distribution coefficients for strontium in calcite determined experimentally by Baker (unpubl. data). The latter were determined in solutions of similar composition and temperature as the pore fluids.

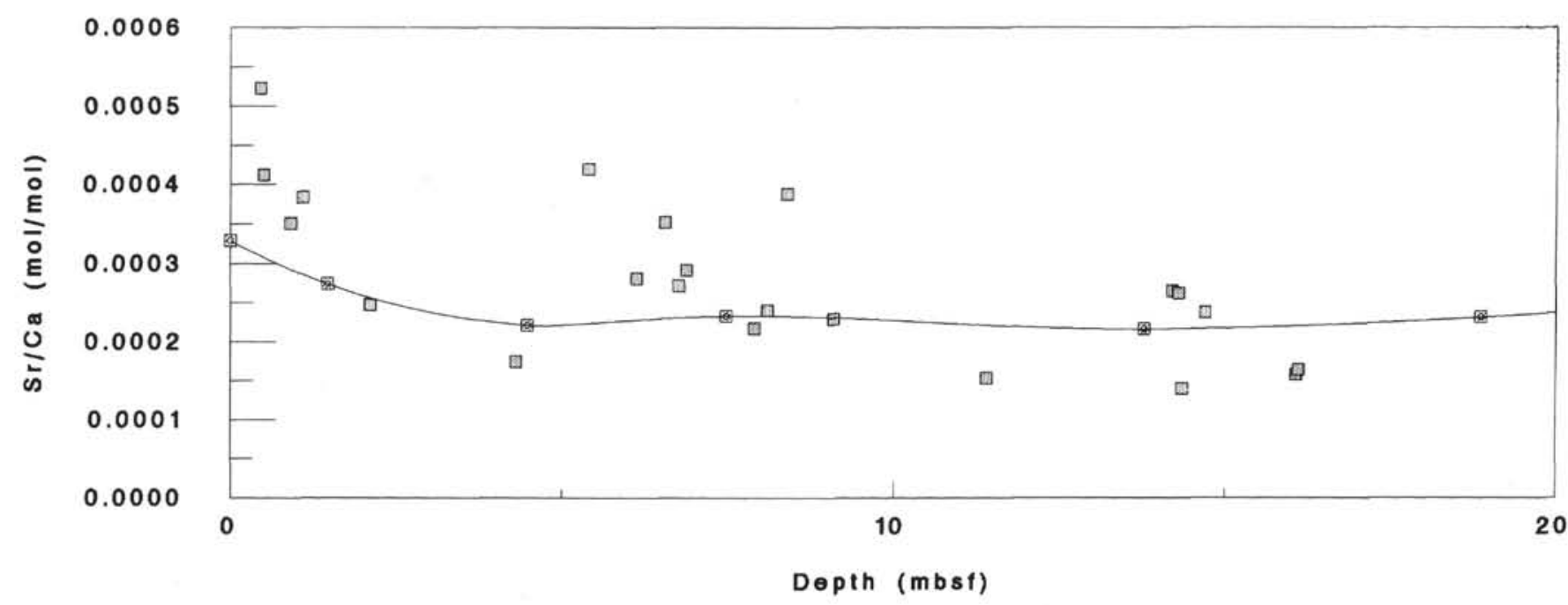

Figure 16. Strontium/calcium ratios of calcites vs. depth for Cores 858D-1H and $-2 \mathrm{H}$. The line is the calculated equilibrium strontium/calcium ratio (see caption for Fig. 15). 


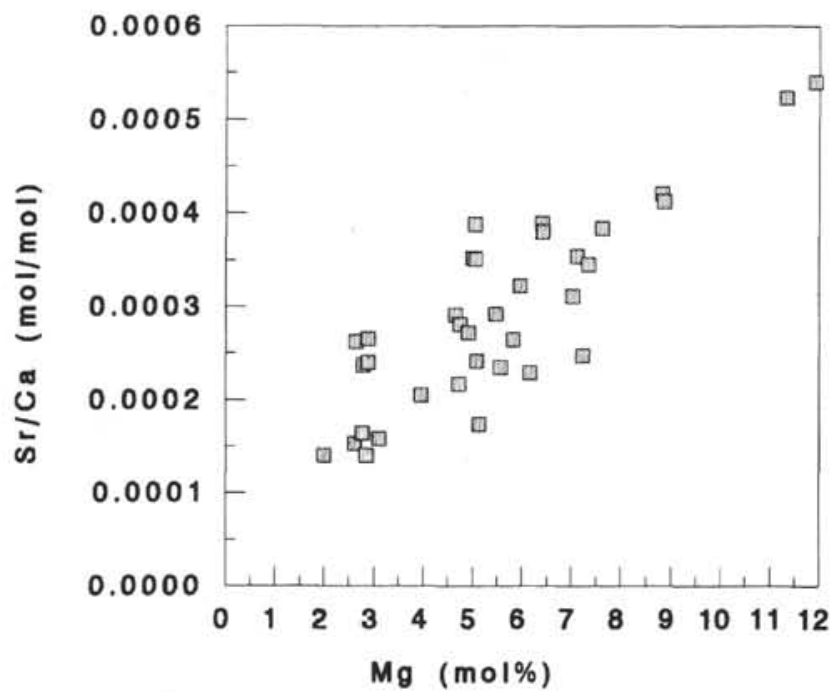

Figure 17. Strontium/calcium ratios of calcite vs, magnesium contents of calcites from Hole 858D.

istry (Wheat and Mottl, this volume) and temperature. There is, however, a good positive correlation $(r=0.85, n=35)$ between the strontium and magnesium contents of the calcites (Fig. 17). This correlation suggests that the strontium contents of calcites in Hole $858 \mathrm{D}$ are controlled by rates of calcite precipitation: high rates of precipitation produce high magnesium contents and also permit high strontium contents (e.g., Mucci and Morse, 1983; Morse and Bender, 1990; Carpenter and Lohmann, 1992). Different controls on the distribution of strontium in calcites from the two holes may be due to more rapid rates of precipitation of calcites in Hole 858D.

\section{MANGANESE AND IRON}

Manganese and iron are abundant substituent ions in the authigenic dolomites and calcites. In Hole $857 \mathrm{C}$, we observe generally higher values of manganese in calcites than in dolomites and higher values of iron in dolomites than in calcites (Table 1). These observa- tions can be rationalized by the ionic radii of these cations which decrease in the order $\mathrm{Ca}>\mathrm{Mn}>\mathrm{Fe}>\mathrm{Mg}$. Hence, manganese substitutes preferentially for calcium in calcite and iron substitutes preferentially for magnesium in dolomite.

There is a general trend toward decreasing values of both iron and manganese in calcites with increasing burial depth in Hole 857C (Fig. 18 ). The trend is amplified when depth is replaced by the oxygen isotopic composition of the carbonate. We speculate that decreasing concentrations of iron and manganese with decreasing $\delta^{18} \mathrm{O}$ are a reflection of the decreased availability of iron and manganese at higher temperatures. Labile iron or manganese, originally associated with the hemipelagic and turbiditic sediments, is released into pore waters in higher quantities during early diagenesis than during deeper burial.

The iron and manganese concentrations in calcites in Hole 858D are more coherent in their behavior and help to clarify the nature of ongoing hydrothermal activity. Figures 19 and 20 show that: (1) iron concentrations in calcites range to a maximum of about two times the concentrations of manganese in calcites; (2) calcites formed at temperatures less than $200^{\circ} \mathrm{C}$ and at burial depths less than $2 \mathrm{mbsf}$ have low concentrations of both iron and manganese; (3) calcites that formed at the highest temperatures (about $200^{\circ} \mathrm{C}$ at about $20 \mathrm{mbsf}$ ) have low concentrations of iron, but relatively high concentrations of manganese; and (4) the highest concentrations of both iron and manganese are found in calcites from about 3 to $7 \mathrm{mbsf}$, the same interval as a pore-water chlorinity maximum (Davis, Mottl, Fisher, et al., 1992). These calcites were precipitated at temperatures between about $30^{\circ}$ and $70^{\circ} \mathrm{C}$.

The plot of calcitic iron and manganese against $\delta^{13} \mathrm{C}$ shows additional separation of these metals during very shallow burial (Fig. 21). Manganese varies linearly with $\delta^{13} \mathrm{C}$ from low concentrations in surficial, methanogenic calcites (shallowest occurrence of $0.46 \mathrm{mbsf}$ ) to high concentrations in deeper calcites (up to $5.40 \mathrm{mbsf}$ ) with a $\delta^{13} \mathrm{C}$ of $-16.97 \%$. The high-manganese calcites are believed to be precipitating in the sedimentary pore waters that most closely resemble the presentday vent fluids; hence, a carbon isotopic value of about $-17 \%$ is believed to be the most characteristic of the hydrothermal source.

The pore-water manganese and iron concentration-depth profiles at Hole $858 \mathrm{D}$ (Wheat and Mottl, this volume) resemble the profiles in the calcites. Pore-water iron is nearly zero at the sediment surface and near the bottom of the core; it attains a maximum value at about $5 \mathrm{mbsf}$. Pore-water manganese is very low in the uppermost sediments, attains a maximum value at about $5 \mathrm{mbsf}$, decreases to a minimum value

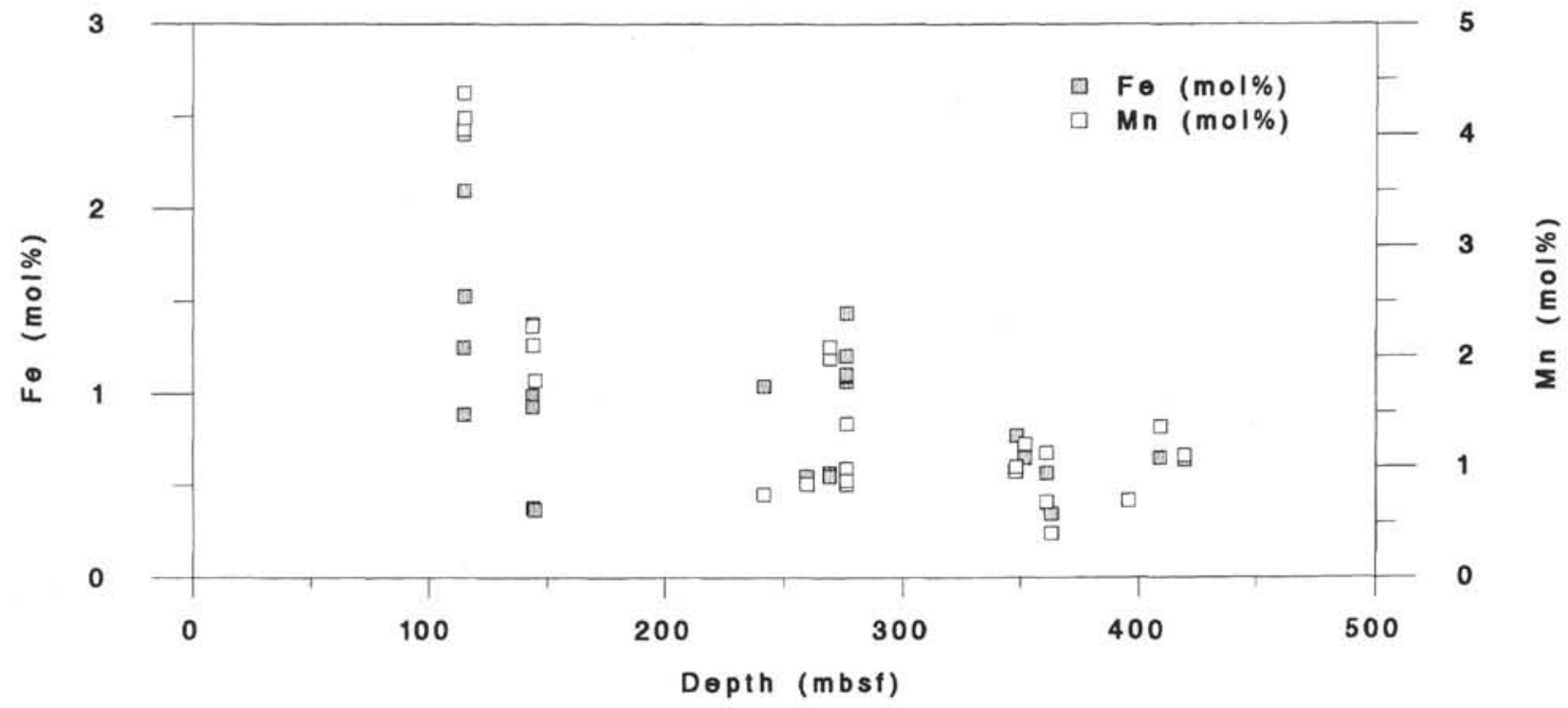

Figure 18. Iron and manganese contents of calcites vs. depth for Hole 857C. 


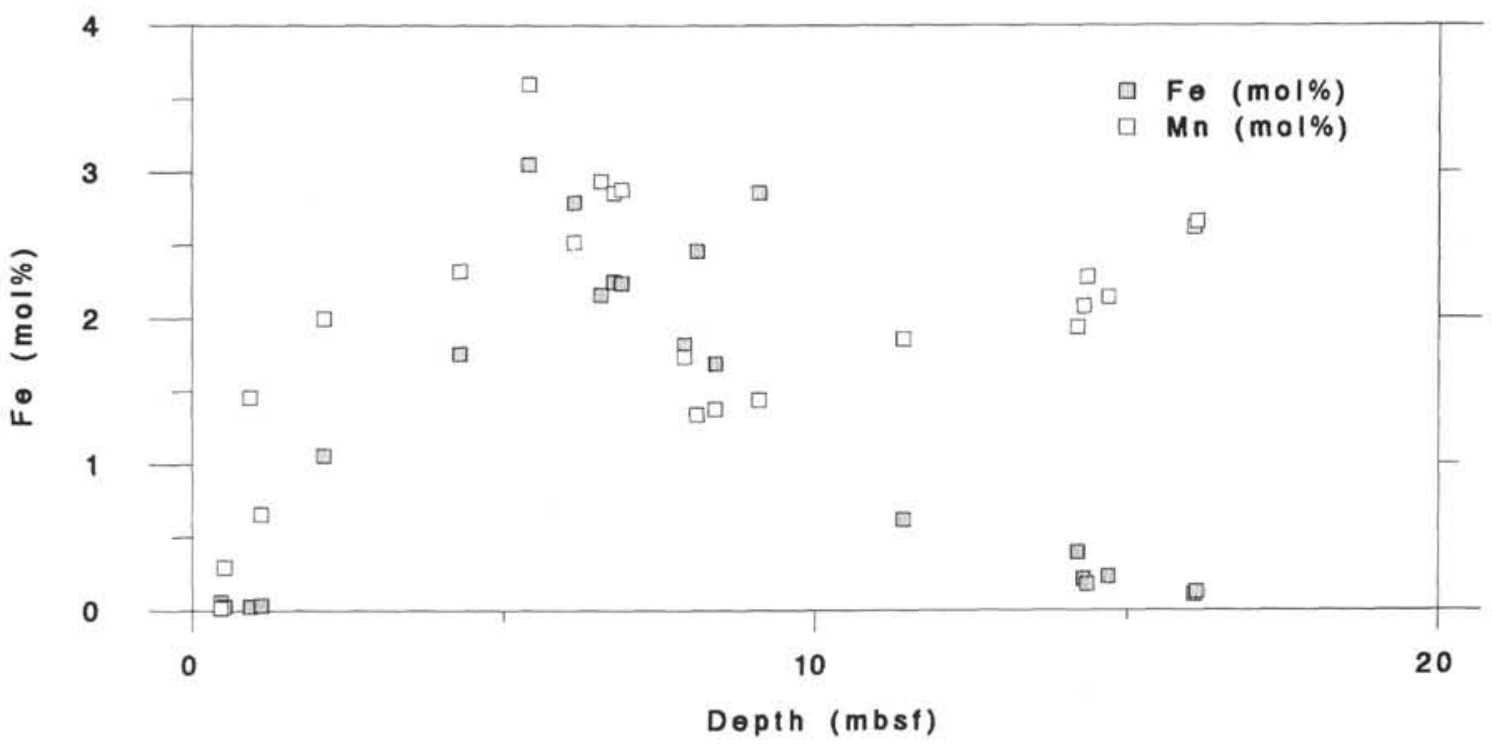

Figure 19. Iron and manganese contents of calcites vs. depth for Cores $858 \mathrm{D}-1 \mathrm{H}$ and $-2 \mathrm{H}$.

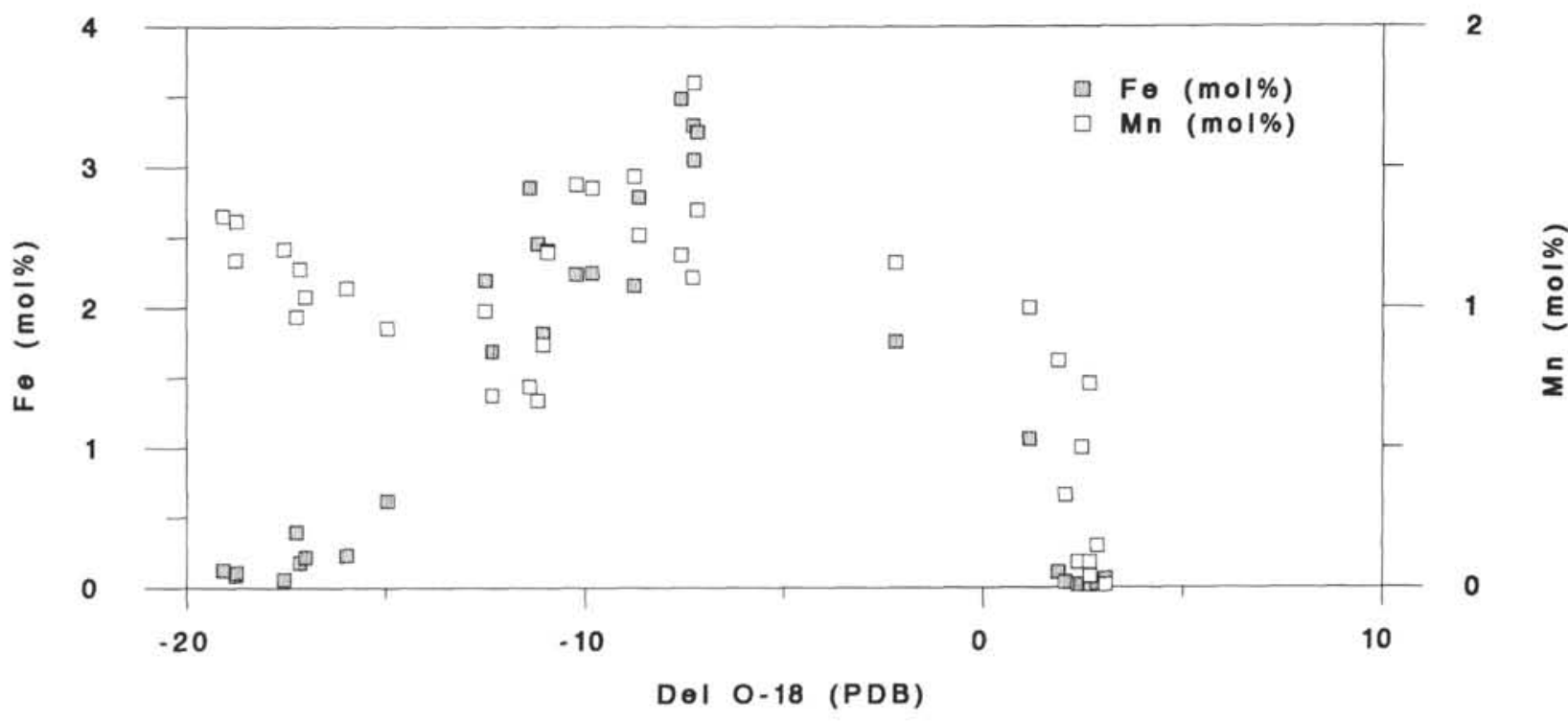

Figure 20. Iron and manganese contents of calcites vs. oxygen isotopic compositions of calcites for Hole 858D.

between 12 and $20 \mathrm{mbsf}$, and then attains an intermediate value near the base of the hole. The pore-water measurements of these metals are quite noisy, however, a likely result of sampling artifacts. Furthermore, pore-water data between 19.8 and 23.04 mbsf are unreliable because, as the isotopic data in this work show, this section is fall-in.

Some of the aforementioned differences between iron and manganese concentrations in calcites are likely due to downhole variability in the concentration of dissolved sulfide. Where dissolved sulfide is high, dissolved iron should be precipitated as iron sulfide and iron concentrations in calcite should be low. Because manganese is not as readily incorporated into sulfide minerals as is iron, the concentration of dissolved sulfide does not significantly affect manganese concentrations in pore fluids or in diagenetic calcites. This is clearly illustrated by calcites from the upper 2 mbsf of Hole 858D. In this depth range microbial sulfate reduction is an active process. Dissolved iron is removed as iron sulfides and little reduced iron is present for incorporation into diagenetic carbonates (Fig. 19). We also believe that at temperatures above $100^{\circ} \mathrm{C}$, thermochemical sulfate reduction by dissolved or gaseous methane (Krouse et al., 1988) is an active process, producing dissolved sulfide. Thus, the shallowest and the deepest pore fluids at Hole $858 \mathrm{D}$ have little dissolved iron and the associated calcites are non-ferroan. These observations have important implications for the shallow circulation of hydrothermal fluids at Site 858 (see Fig. 22). We propose that the maximum values of iron and manganese, coincident with the chlorinity maximum, are due to lateral advection of fluid within a depth range of about 3 to $7 \mathrm{mbsf}$. The lateral flow is not rapid enough, however, to transport significant heat. Heat flow in Hole 858D, as stated previously in this paper, is probably satisfactorily modeled as purely conductive (albeit two- or three-dimensional). Linear extrapolation of the data in Figure 9 to the temperature of present-day vent fluids $\left(276^{\circ} \mathrm{C}\right)$ yields a depth of 28 mbsf. We propose that 28 mbsf is the depth at which Hole $858 \mathrm{D}$ intersects one of the faults that serves as an upward conduit for venting fluids. Additional evidence for this proposal includes poor core re- 


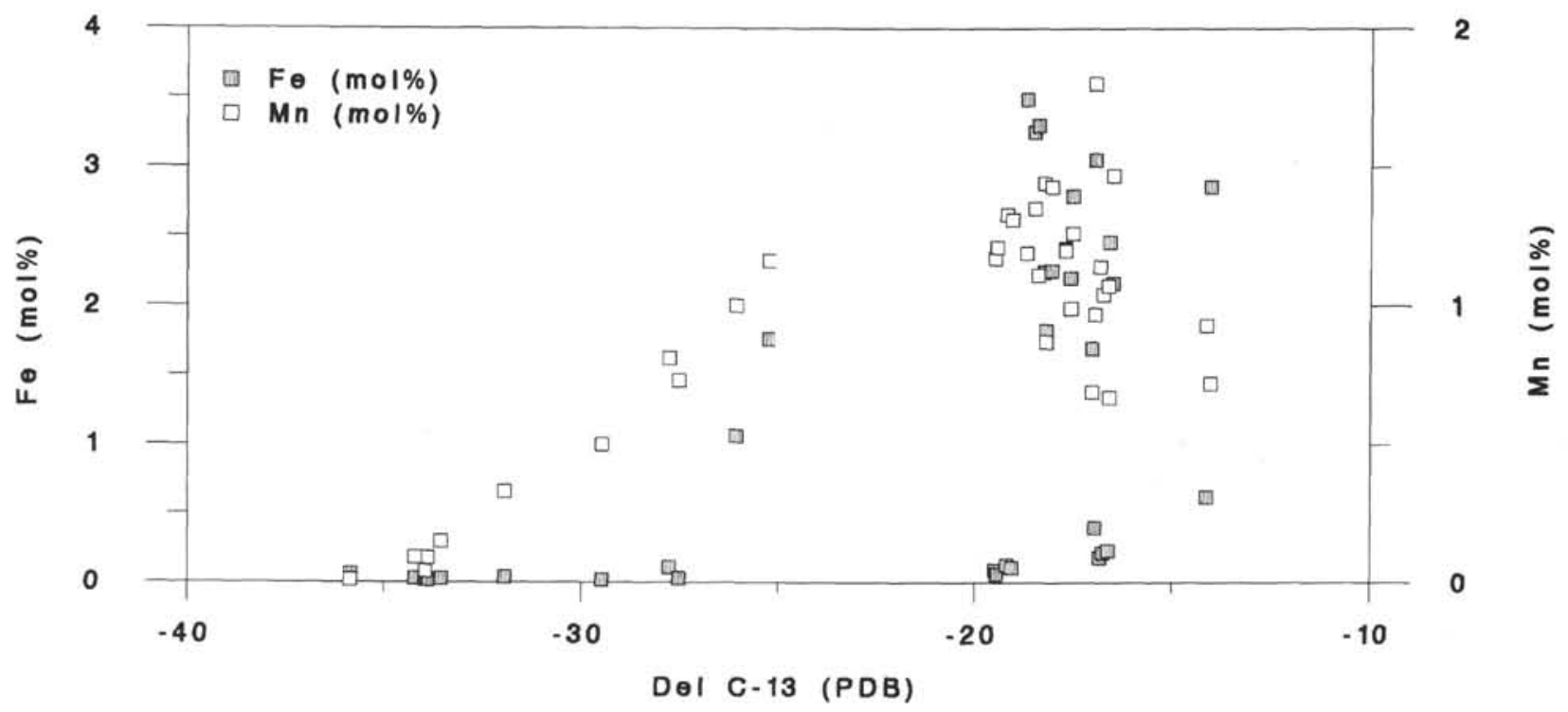

Figure 21. Iron and manganese contents of calcites vs. carbon isotopic compositions of calcites for Hole 858D.

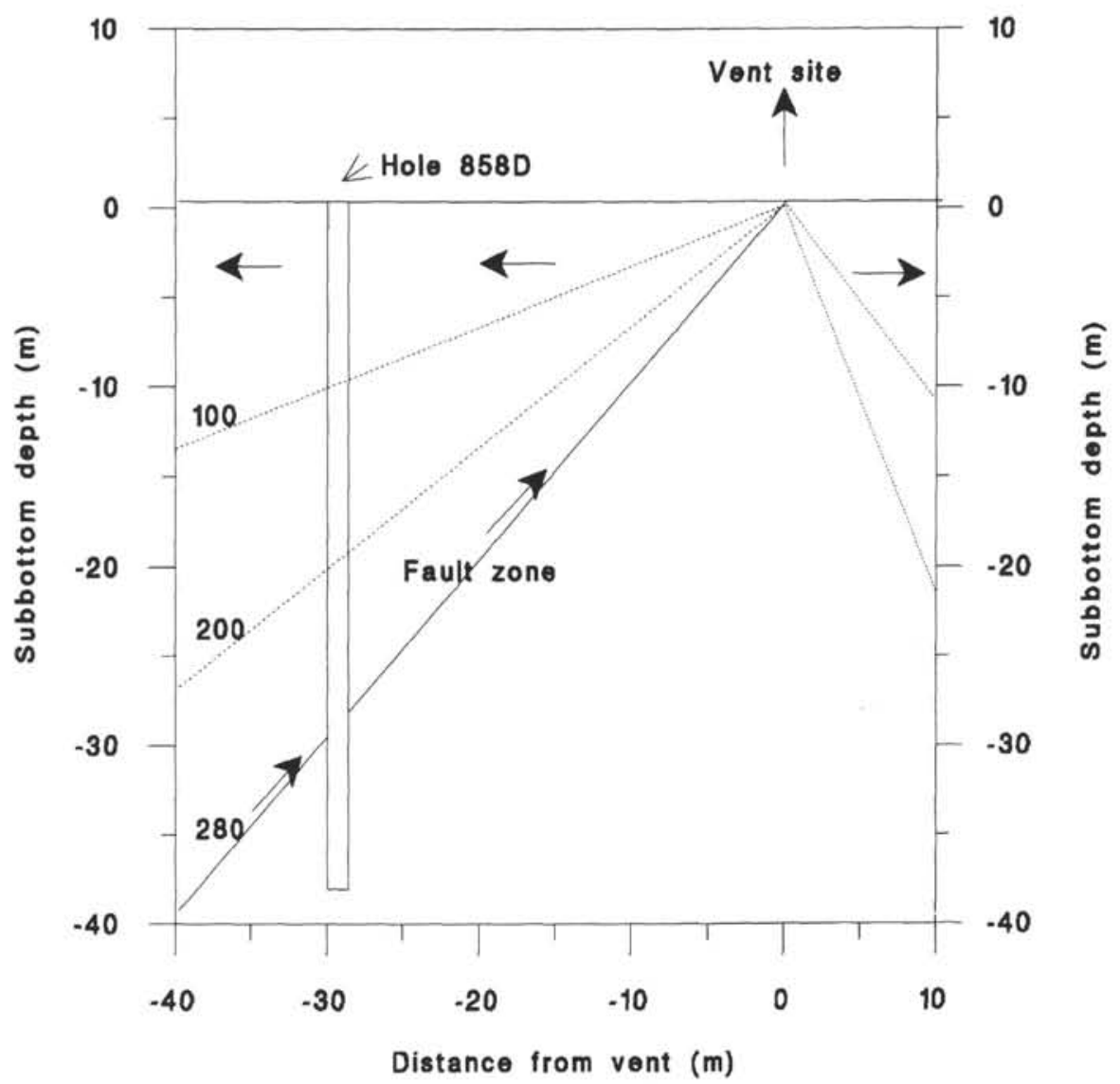

Figure 22. Schematic diagram showing inferred flow paths (arrows), fault zone (solid diagonal line), and isotherms (dashed lines, labelled with temperature, ${ }^{\circ} \mathrm{C}$ ) in the vicinity of Hole 858D.

covery, decreased penetration rate, a large jump in wet-bulk density, and a large decrease in porosity at about $28 \mathrm{mbsf}$ (Davis, Mottl, Fisher, et al., 1992, table 41, p. 540). There is also some evidence that rocks within this depth range are highly silicified (Davis, Mottl, Fisher, et al., 1992, tables 20 and 22). Below 28 mbsf it is likely that thermal gradients decrease.

\section{CONCLUSIONS}

1. Carbonate nodules and cements are abundant throughout the upper portions of the sediment columns in Middle Valley.

2. These carbonates are of a mixed origin: they form partially from the recrystallization of biogenic calcite and partially from the 
direct precipitation of carbonate from pore fluids. In the latter case, calcium ions are sourced from plagioclase alteration in the sediments or basement rocks; magnesium ions (in the dolomites and HMC) are sourced from seawater; and carbon is supplied variously from seawater, from oxidation of sedimentary organic matter, or from oxidation of thermogenic methane. Strontium contained within the carbonates is a mixture of marine-derived (seawater or biogenic calcite) and basement-derived.

3. The carbonates recrystallize relatively rapidly, thus maintaining compositions nearly in thermodynamic and isotopic equilibrium with adjacent pore fluids in sites with prograde histories. In Hole 857C, the calculated thermal gradient is $0.53^{\circ} \mathrm{C} / \mathrm{m}$. In Hole $858 \mathrm{D}$, the calculated thermal gradient is $10.5^{\circ} \mathrm{C} / \mathrm{m}$. In both holes, thermal gradients are consistent with conductive heat transport. In Hole $856 \mathrm{~A}$, the calculated temperatures are much higher than present-day temperatures.

4. We believe the high temperatures are a result of hydrothermal activity associated with sill emplacement and, possibly, formation of the massive sulfide ore body. Because the thermal signature is found in the uppermost sample analyzed in Hole 856A from $14.5 \mathrm{mbsf}$, we believe the event was recent, perhaps within the last 10,000 or 20,000 years. In Hole $858 \mathrm{~A}$, somewhat higher than present-day temperatures are also recorded by several samples.

5. We believe that Hole $858 \mathrm{D}$ penetrated through a fault zone at about 28 mbsf. This fault zone is a high-permeability conduit for the hydrothermal vent fluids that discharge a few tens of meters away. The fault zone is silicified, perhaps because of depressurization, temperature drop, $\mathrm{pH}$ increase, or salinity decrease during the rapid ascent of hydrothermal fluid and mixing with neighboring pore fluids. Although most of the hydrothermal fluid is discharged at the seafloor, some of it advects laterally through the sediments. In Hole 858D this lateral flow takes place at a depth of about 3 to 7 mbsf. Maximum iron and manganese concentrations are found in calcites precipitated within this depth range.

\section{ACKNOWLEDGMENTS}

This work would not have been possible without the help of the entire crew and scientific party of the JOIDES Resolution. We also thank the shore-based personnel at ODP in College Station for their efforts on our behalf. This paper has benefitted from the reviews of John Compton, Mike Mottl, an anonymous reviewer, and the ODP editorial staff. Funding for this work was provided by JOI/USSSP. This support was invaluable. We are also grateful to Ms. Debbie Gooch for her help in preparation of the manuscript.

\section{REFERENCES*}

Al-Aasm, LS., and Blaise, B., 1991. Interaction between hemipelagic sediment and a hydrothermal system: Middle Valley, northern Juan de Fuca Ridge, subarctic northeast Pacific. Mar. Geol., 98:25-40.

Baker, P.A., and Burns, S.J., 1985. The occurrence and formation of dolomite in organic-rich continental margin sediments. AAPG Bull., 69:1917-1930.

Carpenter, S.J., and Lohmann, K.C., 1992. Sr/Mg ratios of modern marine calcite: empirical indicators of ocean chemistry and precipitation rate. Geochim. Cosmochim. Acta, 56:1837-1849.
Claypool, G.E., and Kaplan, I.R., 1974. The origin and distribution of methane in marine sediments. In Kaplan, I.R. (Ed.), Natural Gases in Marine Sediments: New York (Plenum), 99-139.

Davis, E.E., Mottl, M.J., Fisher, A.T., et al., 1992. Proc. ODP, Init. Repts., 139: College Station, TX (Ocean Drilling Program).

Friedman, I., and O'Neil, J.R., 1977. Compilation of stable isotope fractionation factors of geochemical interest. In Fleischer, M. (Ed.), Data of Geochemistry (6th Ed.). Geol. Surv. Prof. Pap. U.S., 440-KK:1-12.

Fritz, P., and Smith, D.C.W., 1970. The isotopic composition of secondary dolomites. Geochim. Cosmochim. Acta., 34:1161-1173.

Goodfellow, W.D., and Blaise, B., 1988. Sulfide formation and hydrothermal alteration of hemipelagic sediment in Middle Valley, northern Juan de Fuca Ridge. Can. Mineral., 26:675-696.

Horwitz, E., Dietz, M., and Fisher, D., 1991. Separation and preconcentration of strontium from biological, environmental and nuclear waste samples by extraction chromatography using a crown ether. Anal. Chem., 63:522-525.

Kelts, K., and McKenzie, J.A., 1982. Diagenetic dolomite formation in Quaternary anoxic diatomaceous muds of Deep Sea Drilling Project Leg 64, Gulf of California. In Curray, J.R., Moore, D.G., et al., Init. Repts. DSDP, 64 (Pt. 2): Washington (U.S. Govt. Printing Office), 553-569.

Krouse, H.R., Viau, C.A., Elink, L.S., Ueda, A., and Halas, S., 1988. Chemical and isotopic evidence of thermochemical sulphate reduction by light hydrocarbon gases in deep carbonate reservoirs. Nature, 333:415-519.

Land, L.S., 1987. The major ion chemistry of saline brines in sedimentary basins. Am. Inst. Phys. Conf. Proc., 154:160-179.

Lorens, R.B., 1981. Sr, Cd, Mn and Co distribution coefficients in calcite as a function of calcite precipitation rate. Geochim. Cosmochim. Acta., 45:553561.

McCrea, J.M., 1950. Isotopic chemistry of carbonates and a paleotemperature scale. J. Chem. Phys., 18:849-857.

Mook, W.G., Bommerson, J.C., and Staverman, W.H., 1974. Carbon isotope fractionation between dissolved bicarbonate and gaseous carbon dioxide. Earth Planet. Sci. Lett., 22:169-176.

Morse, J.W., and Bender, M.L., 1990. Partition coefficients in calcite: examination of factors influencing the validity of experimental results and their application to natural systems. Chem. Geol., 82:265-277.

Mucci, A., and Morse, J.W., 1983. The incorporation of $\mathrm{Mg}^{2+}$ and $\mathrm{Sr}^{2+}$ into calcite overgrowths: influence of growth rate and solution composition. Geochim. Cosmochim. Acta, 47:217-233.

O'Neil, J.R., Clayton, R.N., and Mayeda, T.K., 1969. Oxygen isotope fractionation in divalent metal carbonates. J. Chem. Phys., 51:5547-5558.

Rosenberg, P.E., Burt, D.M., and Holland, H.D., 1967. Calcite-dolomite-magnesite stability relations in solutions: the effect of ionic strength. Geochim. Cosmochim. Acta, 31:391-396.

Sheppard, S.M.F., and Schwarcz, H.P., 1970. Fractionation of carbon and oxygen isotopes and magnesium between coexisting metamorphic calcite and dolomite. Contrib. Mineral. Petrol., 26:161-198.

\footnotetext{
-Abbreviations for names of organizations and publications in ODP reference lists follow the style given in Chemical Abstracts Service Source Index (published by American Chemical Society).
}

Date of initial receipt: 3 March 1993

Date of acceptance: 1 October 1993

Ms 139SR-215 